\title{
Determination of wheel-rail contact points with semianalytic methods
}

\author{
Monica Malvezzi • Enrico Meli • Stefano Falomi • \\ Andrea Rindi
}

Received: 22 November 2007 / Accepted: 22 July 2008 / Published online: 12 September 2008

(C) Springer Science+Business Media B.V. 2008

\begin{abstract}
The multibody simulation of railway vehicle dynamics needs a reliable and efficient method to determine the location of the contact points between wheel and rail that represent the application points of the contact forces and influence their directions and intensities. In this work, two semi-analytic procedures for the detection of the wheel-rail contact points (named the DIST and the DIFF methods) are presented. Both the methods consider the wheel and the rail as two surfaces whose analytic expressions are known. The first method is based on the idea that the contact points are located in the point in which the distance between the contact surfaces has local maxima, and is equivalent to solve an algebraic $4 \mathrm{D}$-system. The second method is based on the idea that in the contact points the difference between the surfaces has local minima and is equivalent to solve an algebraic $2 \mathrm{D}$-system. In both cases, the original problem can be reduced analytically to a simple 1D-problem that can be easily solved numerically.
\end{abstract}

Keywords Contact point location · Railway simulation · On-line contact detection

\section{Introduction}

The research of the contact points between the wheels and the rails in the multibody simulation of railway vehicle dynamics is very important since they represent the application points of the contact forces. The wheel/rail contact problem has been discussed by several authors and a number of procedures to evaluate the contact points can be found in the literature.

M. Malvezzi $(\varangle) \cdot$ E. Meli · S. Falomi · A. Rindi

Department of Energetics, University of Florence, via S. Marta n. 3, 50139 Firenze, Italy

e-mail: malvezzi@mapp1.de.unifi.it

E. Meli

e-mail: meli@mapp1.de.unifi.it

S. Falomi

e-mail: falomi@mapp1.de.unifi.it

A. Rindi

e-mail: rindi@mapp1.de.unifi.it 
In the simulation of the railway dynamics, there are two different approaches to solve the wheel/rail contact problem: the so-called rigid contact formulation [1] and the semielastic contact description [2, 3]. In the rigid approach (the classical multibody approach), the contact is represented by means of the constraint equations that impose the contact between the wheel and the rail surfaces. The contact points are searched during the dynamic simulation by solving the nonlinear algebraic-differential equations associated to the constrained multibody system. In this case, each wheel has only five degrees of freedom with respect to the rail, and the indentation and the lift between the contact bodies are not permitted. This approach has been applied both in the railway [1, 4-6] and in the automotive dynamics [7].

In the formulations based on the elastic approach, the wheel is represented as a rigid body with six degrees of freedom with respect to the rail and the normal contact forces are defined as the function of the indentation between the bodies through the Hertz contact theory $[2,3]$. In the literature, several methods for the detection of the contact points can be found. Some authors started from the idea of minimizing the distance between the wheel and rail surfaces; most of them, however, introduced additional hypotheses on the position of the contact points in order to simplify the geometry of the problem $[6,8,9]$.

In some preceding works, the authors presented a method $[10,11]$ in which the contact points are searched minimizing the difference between the wheel and rail surfaces by means of numerical iterative algorithms (like the Compass Search algorithm and the Simplex algorithm $[12,13])$. These procedures do not introduce additional geometric hypotheses and allow an efficient management of the multiple contacts (up to two contact points for wheel). The challenge of this study was the realization of an efficient multibody model, running in real-time conditions; however, the developed solutions did not allow a direct implementation of the research procedure in the multibody model. In other words, the solutions described in $[10,11]$ were used to generate lookup tables to be used during the simulation of the vehicle dynamics. The wheel/rail contact problem can be solved in this case finding the minima of a two dimensional surface. The numerical algorithms conventionally used for this type of application present various problems:

1. Iterative research algorithms need start points and break conditions that are not simple to choose and may affect the reliability of the solution; moreover, the convergence of the procedure cannot be easily assured.

2. The required accuracy cannot be guaranteed a priori.

3. The management of the multiple solutions can be difficult.

4. The required computational burden allows only off-line implementation (a real time implementation requires necessarily the use of lookup tables).

In this work, the authors propose two semianalytic methods to determine the wheel-rail contact points. These procedures will allow to improve the performances of the preceding methods and to overcome the problems mentioned above. Both the presented procedures represent the wheel and the rail as two mathematical surfaces whose analytic expression is known (Fig. 1). The first method is based on the idea that the contact points are located in the points in which the distance between the wheel surface and the rail surface assumes local maxima. This method has been used by several authors to solve the wheel/rail problem $[2,3,6]$. In the wheel/rail case, the problem can be represented as the research of the stationary point on a four dimensional surface, that is equivalent to solve an algebraic system having the form:

$$
\mathbf{F}_{1}(\mathbf{x})=\mathbf{0}, \quad \mathbf{F}_{1}: \mathbb{R}^{4} \rightarrow \mathbb{R}^{4} .
$$

The second method is based on the idea that in the contact points the difference between the wheel surfaces and the rail surface evaluated along a direction assumes local minima. In this 
Fig. 1 Wheel and rail surfaces

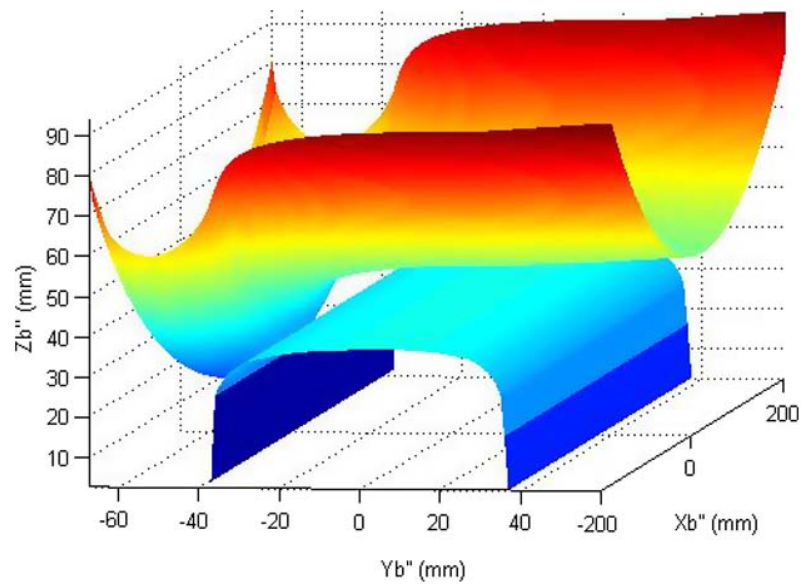

case, the problem can be reduced to the research of the minima of a two dimensional surface and is equivalent to solve an algebraic system that can be written as:

$$
\mathbf{F}_{2}(\mathbf{x})=\mathbf{0}, \quad \mathbf{F}_{2}: \mathbb{R}^{2} \rightarrow \mathbb{R}^{2} .
$$

In both cases, the problem can be reduced analytically to a simple mono-dimensional problem having the form

$$
F(x)=0, \quad F: \mathbb{R} \rightarrow \mathbb{R}
$$

that can be solved numerically. The first method will be indicated in the paper with the abbreviation DIST (Distance Method), whereas the second with DIFF (Difference Method).

The performances of the proposed procedures will be compared among them and with those obtained with other methods previously developed and available in the literature (like the Compass Search and the Simplex methods [10-13]). The results will be also compared with those obtained with the direct research of the solution obtained by the calculation of the function on a multi-dimensional grid and the comparison between the obtained values (this procedure will be indicated with GRID method throughout the paper). The comparisons will be carried out in terms of precision and computation times.

The paper is organized as follows: In Sect. 2, the analytic formulation of the problem will be introduced, in the Sects. 3 and 4, the DIST and DIFF methods will be described in detail; in Sect. 5 the obtained results are described and the performances of the new procedures will be compared with those obtained with other methods. A particular attention will be dedicated to the computation times and the precision of the procedures.

\section{The analytic formulation of the problem}

In this chapter, the mathematical notations used in the paper and the analytic formulation of the problem will be introduced. Firstly, a fixed global reference system $O_{f} x_{f} y_{f} z_{f}$ (Fig. 2) is defined, the $x_{f}$ axis is tangent to the track centerline in the point $O_{f}$ and the $z_{f}$ axis is normal to the plane of the rails. With respect to this fixed global system, the railway track can be described by means of a three-dimensional curve $\Gamma(s)$ ( $s$ is the curvilinear abscissa):

$$
\Gamma: I \subset \mathbb{R} \rightarrow \mathbb{R}^{3} .
$$


Fig. 2 Definition of the rail track, base, and auxiliary reference systems

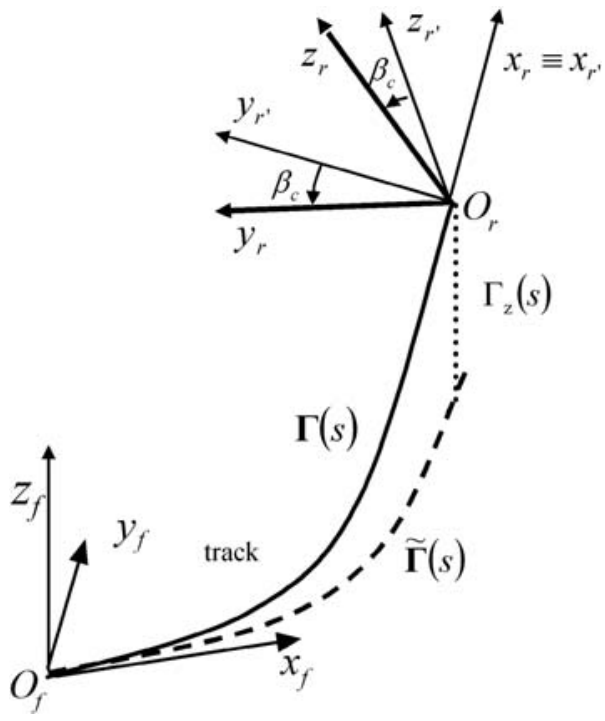

For convenience, $\tilde{\boldsymbol{\Gamma}}(s)$ is defined as the projection of $\boldsymbol{\Gamma}(s)$ on the horizontal plane $x_{f} y_{f}$. Usually in the cartographic description of a railway track, the profile is described by its projection on the horizontal plane and by its longitudinal profile. The curvature $K(s)$ of the projection $\tilde{\boldsymbol{\Gamma}}(s)$ can be obtained from the track planimetry, while the track slope $p(s)$ that represents the tangent of the angle between the track and the horizontal plane can be obtained from the longitudinal track profile [15]. From these parameters, the three-dimensional track curve $\Gamma(s)$ can be calculated. The track is usually composed of a series of straight, circular, or transition curve segments and their analytical expressions are known. In the more general case, the curve profile can be obtained integrating the Frenet equations [14]:

$$
\frac{d \mathbf{t}}{d s}=K(s) \mathbf{n}(s), \quad \frac{d \mathbf{n}}{d s}=-K(s) \mathbf{t}(s),
$$

where $\mathbf{t}(s)$ and $\mathbf{n}(s)$ are respectively the tangent and the normal unitary vectors of the curve $\tilde{\boldsymbol{\Gamma}}(s)$. Starting from the boundary conditions $\mathbf{t}(0)=[1,0]^{T}$ and $\mathbf{n}(0)=[0,1]^{T}$ (in this configuration, the tangent unitary vector is parallel to $x_{f}$ and the normal one is parallel to $y_{f}$ ), the Frenet equations can be integrated, obtaining $\mathbf{t}(s)$ and $\mathbf{n}(s)$. Then since $\mathbf{t}(s)=\frac{\tilde{\boldsymbol{\Gamma}}(s)}{d s}$, the first two components of $\boldsymbol{\Gamma}(s)$ can be calculated by the following integration:

$$
\tilde{\boldsymbol{\Gamma}}(s)=\int_{0}^{s} \mathbf{t}(u) d u,
$$

in which $u$ represents the integration variable. The third component of $\boldsymbol{\Gamma}(s)$ is calculated using the definition of the track slope:

$$
\Gamma_{z}(s)=\int_{0}^{s} p(u) d u .
$$

A second reference system (referred as auxiliary reference system) $O_{r} x_{r} y_{r} z_{r}$ (Fig. 3) is defined, $\mathbf{i}_{r}, \mathbf{j}_{r}$, and $\mathbf{k}_{r}$ represent the unitary vectors relative to the axes $x_{r}, y_{r}$, and $z_{r}$, respectively. It is defined on the rails, but follows the wheelset during the simulation. The $x_{r}$ axis 
Fig. 3 Track auxiliary reference system and wheelset local reference systems

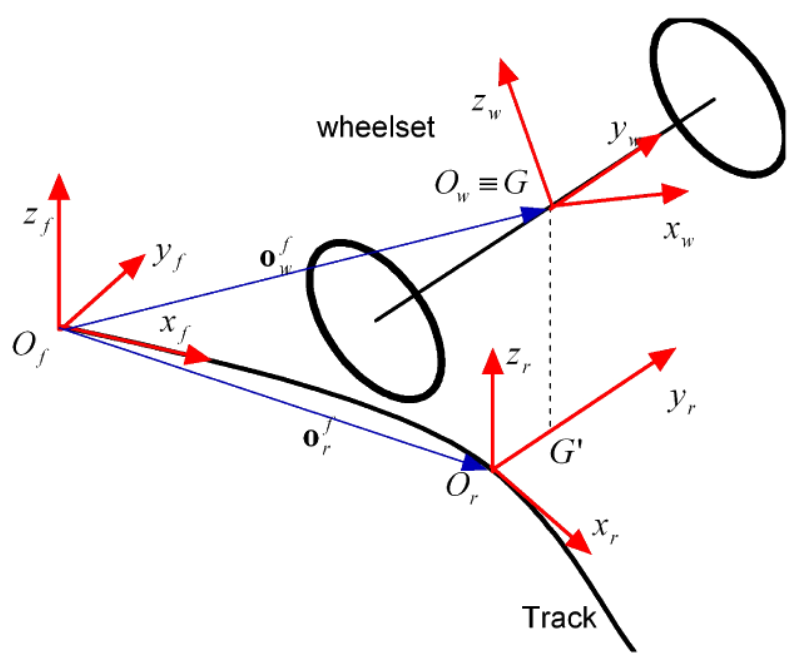

is tangent to the track centerline in the point $O_{r}$ and the $z_{r}$ axis normal to the plane of the rails. The position of the point $O_{r}$, identified by its coordinates $\mathbf{o}_{r}^{f}$ relative to the fixed reference system, can be calculated from the wheelset center of mass location $G$, identified by its coordinates relative to the fixed reference system $\mathbf{o}_{w}^{f}$, imposing the following condition:

$$
\left(\mathbf{o}_{w}^{f}-\mathbf{o}_{r}^{f}\right) \cdot \mathbf{i}_{r}=0,
$$

since the point $O_{r}$ is on the curve $\boldsymbol{\Gamma}(s)$ describing the rail track, $\mathbf{o}_{r}^{f}=\boldsymbol{\Gamma}(s)$ and $\mathbf{i}_{r}=\mathbf{i}_{r}(s)$, then (8) can be rewritten as:

$$
\left(\mathbf{o}_{w}^{f}-\boldsymbol{\Gamma}(s)\right) \cdot \mathbf{i}_{r}(s)=0 .
$$

Equation (9) can be solved with respect to the variable $s$. This condition is equivalent to impose that the plane $y_{r} z_{r}$ contains the wheelset center of mass $G$.

In order to define the axes $y_{r}$ and $z_{r}$, another reference system (named secondary reference system) is defined, its unitary vectors $\mathbf{i}_{r^{\prime}}, \mathbf{i}_{r^{\prime}}$, and $\mathbf{i}_{r^{\prime}}$, relative to the axes $x_{r^{\prime}}, y_{r^{\prime}}$, and $z_{r^{\prime}}$, respectively, are calculated as follows:

$$
\begin{aligned}
\mathbf{i}_{r^{\prime}} & =\mathbf{i}_{r}=\frac{d \boldsymbol{\Gamma}}{d s} /\left\|\frac{d \boldsymbol{\Gamma}}{d s}\right\|, \\
\mathbf{j}_{r^{\prime}} & =\mathbf{k}_{f} \times \mathbf{i}_{r^{\prime}}, \\
\mathbf{k}_{r^{\prime}} & =\mathbf{i}_{r^{\prime}} \times \mathbf{j}_{r^{\prime}} .
\end{aligned}
$$

The unitary vectors of the auxiliary system can then be defined as follows:

$$
\left[\begin{array}{lll}
\mathbf{i}_{r} & \mathbf{j}_{r} & \mathbf{k}_{r}
\end{array}\right]=\left[\begin{array}{lll}
\mathbf{R}_{\mathrm{cant}}
\end{array}\right]\left[\begin{array}{lll}
\mathbf{i}_{r^{\prime}} & \mathbf{j}_{r^{\prime}} & \mathbf{k}_{r^{\prime}}
\end{array}\right]=\left[\mathbf{R}_{1}\right],
$$

where the rotation matrix $\left[\mathbf{R}_{\text {cant }}\right]$ is defined as:

$$
\left[\mathbf{R}_{\mathrm{cant}}\right]=\left[\mathbf{R}_{x, \beta_{\mathrm{c}}}\right]=\left[\begin{array}{ccc}
1 & 0 & 0 \\
0 & \cos \beta_{\mathrm{c}} & -\sin \beta_{\mathrm{c}} \\
0 & \sin \beta_{\mathrm{c}} & \cos \beta_{\mathrm{c}}
\end{array}\right],
$$


$\beta_{\mathrm{c}}$ is the cant angle and $\left[\mathbf{R}_{1}\right]$ is the rotation matrix that links the auxiliary system with the fixed one.

Finally, the local reference system $O_{w} x_{w} y_{w} z_{w}$ is defined. The $y_{w}$ axis is coincident with the rotation axis of the wheels and is rigidly connected to the axle (except for the rotation around this axis). The $x_{w}$ axis is parallel to the plane $x_{r} y_{r}$ and the origin $O_{w}$ coincides with the center of mass $G$ of the wheelset.

Indicating with $\mathbf{p}^{f}, \mathbf{p}^{r}$, and $\mathbf{p}^{w}$ the position of a generic point expressed respectively in the fixed, auxiliary, and local reference systems, then the following standard kinematic relations hold:

$$
\begin{aligned}
\mathbf{p}^{f} & =\mathbf{o}_{r}^{f}+\left[\mathbf{R}_{1}\right] \mathbf{p}^{r}, \\
\mathbf{p}^{r} & =\mathbf{o}_{w}^{r}+\left[\mathbf{R}_{2}\right] \mathbf{p}^{w}, \\
\mathbf{p}^{f} & =\mathbf{o}_{w}^{f}+[\widehat{\mathbf{R}}] \mathbf{p}^{w},
\end{aligned}
$$

where $[\widehat{\mathbf{R}}]=\left[\mathbf{R}_{1}\right]\left[\mathbf{R}_{2}\right]$ is the rotation matrix that links the local system with the fixed one, $\mathbf{o}_{w}^{r}$ and $\mathbf{o}_{w}^{f}$ are the coordinates of the wheelset center of mass expressed in the auxiliary and in the fixed reference system, respectively. The matrix $\left[\mathbf{R}_{2}\right]$ (that links the local system with the auxiliary one) is defined as:

$$
\begin{aligned}
{\left[\mathbf{R}_{2}\right] } & =\left[\mathbf{R}_{z, \alpha}\right]\left[\mathbf{R}_{x, \beta}\right]=\left[\begin{array}{ccc}
\cos \alpha & -\sin \alpha & 0 \\
\sin \alpha & \cos \alpha & 0 \\
0 & 0 & 1
\end{array}\right]\left[\begin{array}{ccc}
1 & 0 & 0 \\
0 & \cos \beta & -\sin \beta \\
0 & \sin \beta & \cos \beta
\end{array}\right] \\
& =\left[\begin{array}{ccc}
\cos \alpha & -\sin \alpha \cos \beta & \sin \alpha \sin \beta \\
\sin \alpha & \cos \alpha \cos \beta & -\cos \alpha \sin \beta \\
0 & \sin \beta & \cos \beta
\end{array}\right],
\end{aligned}
$$

where $\alpha$ and $\beta$ are respectively the yaw and roll angles of the axle with respect to the track.

In order to parametrize a surface, different options can be considered. However, being the wheel a solid of revolution, an angular parameter, and a translational parameter in the direction of the wheel profile seem the natural option. Similarly, an arc length parameter and a translational parameter in the direction of the rail profile is also a natural option for the track. However, in this work for both wheel and rail, the surfaces two translational parameters have been used; this choice allows to simplify the algebraic manipulations necessary to develop the DIST and DIFF methods described in the following sections.

In the local system the axle (and, therefore, the wheels) can be described by means of a revolution surface. The generative function, schematically sketched in Fig. 4 is indicated with $r\left(y_{w}\right)$ (the function $r\left(y_{w}\right)$ is known). The profile of the single wheel is plotted in detail in Fig. 5. In this case, an ORE S 1002 has been chosen [15]. The position of a generic point of the axle in the local reference frame $\mathbf{p}_{w}^{w}$ has consequently the following analytic expression (Fig. 6):

$$
\mathbf{p}_{w}^{w}\left(x_{w}, y_{w}\right)=\left[\begin{array}{c}
x_{w} \\
y_{w} \\
-\sqrt{r\left(y_{w}\right)^{2}-x_{w}^{2}}
\end{array}\right],
$$


Fig. 4 Generative function of the wheelset

Fig. 5 Profile of the wheel

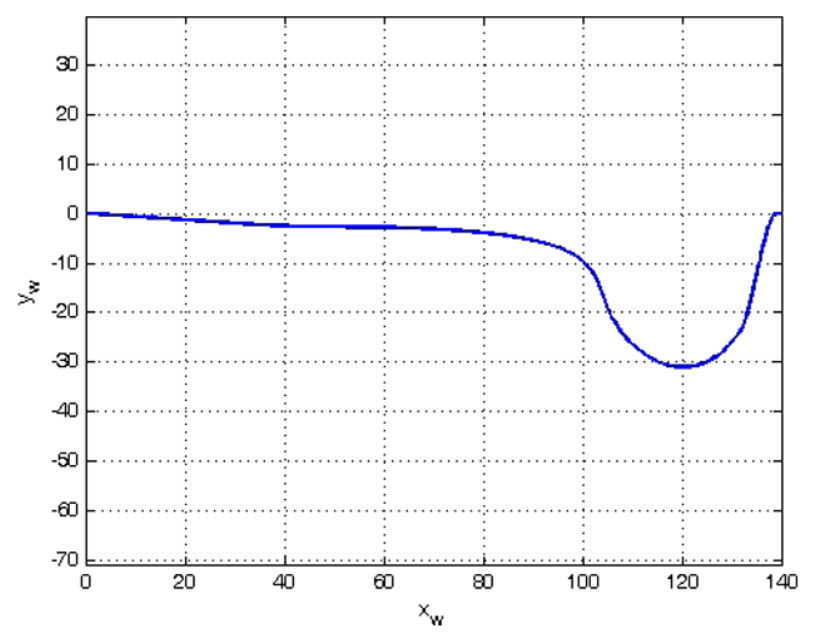

while the position of the same point in the auxiliary system is given by (Fig. 3):

$$
\mathbf{p}_{w}^{r}\left(x_{w}, y_{w}\right)=\mathbf{o}_{w}^{r}+\left[\mathbf{R}_{2}\right] \mathbf{p}_{w}^{w}\left(x_{w}, y_{w}\right)=\left[\begin{array}{c}
x_{w}^{r}\left(x_{w}, y_{w}\right) \\
y_{w}^{r}\left(x_{w}, y_{w}\right) \\
z_{w}^{r}\left(x_{w}, y_{w}\right)
\end{array}\right]
$$

as consequence of the choice of the reference systems the matrix $\left[\mathbf{R}_{2}\right]$, defined in (14) as a function of the wheelset yaw and roll angle has the following structure:

$$
\left[\mathbf{R}_{2}\right]=\left[\begin{array}{c}
\mathbf{r}_{1}^{T} \\
\mathbf{r}_{2}^{T} \\
\mathbf{r}_{3}^{T}
\end{array}\right]=\left[\begin{array}{ccc}
r_{11} & r_{12} & r_{13} \\
r_{21} & r_{22} & r_{23} \\
0 & r_{32} & r_{33}
\end{array}\right]
$$

while the coordinates of the wheelset center of mass are:

$$
\mathbf{o}_{w}^{r} b=\left[\begin{array}{c}
0 \\
G_{y} \\
G_{z}
\end{array}\right] .
$$




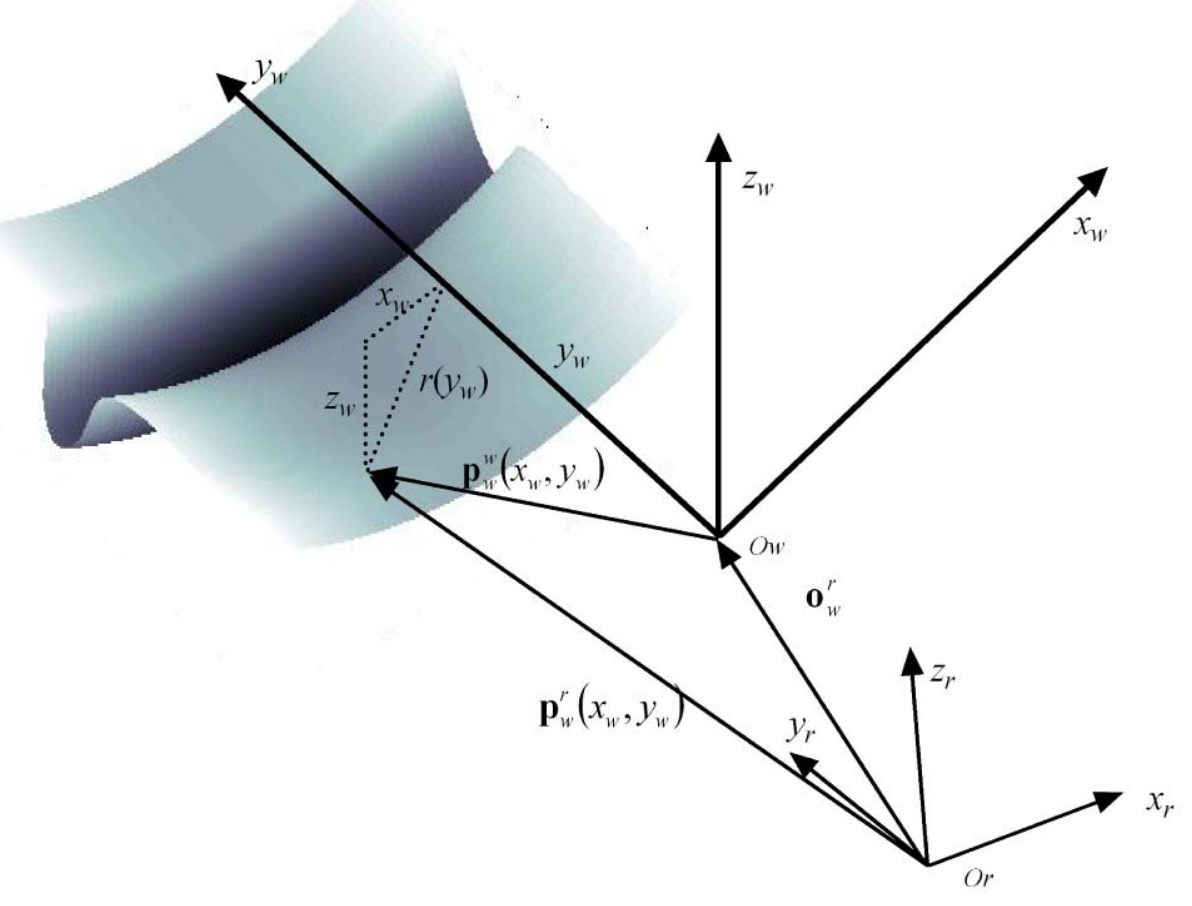

Fig. 6 Coordinates of a point on the wheel surface

Fig. 7 Railway generative function

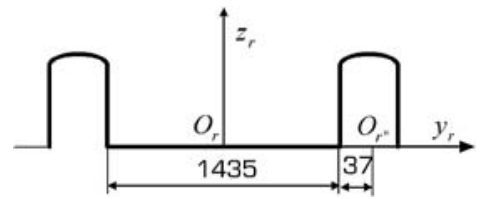

Similarly, the rails can be described in the auxiliary system by means of a extrusion surface. The generative function indicated with $b\left(y_{r}\right)$ is known and is sketched in Fig. 7.

The profile of the single rail is plotted in detail in Fig. 8. This profile is rotated with respect to the $x_{r}$ axis with an angle $\alpha_{p}$ corresponding to the railway laying angle, in the figure, an UIC 60 is shown [15].

The position of a generic point of the rail in the auxiliary system are:

$$
\mathbf{p}_{r}^{r}\left(x_{r}, y_{r}\right)=\left(\begin{array}{c}
x_{r} \\
y_{r} \\
b\left(y_{r}\right)
\end{array}\right) .
$$

For both surfaces the normal unitary vectors (outgoing for convention) can be defined. The normal unitary vector on the wheel surface (Fig. 9) is defined, in the local system, 
Fig. 8 Rail profile

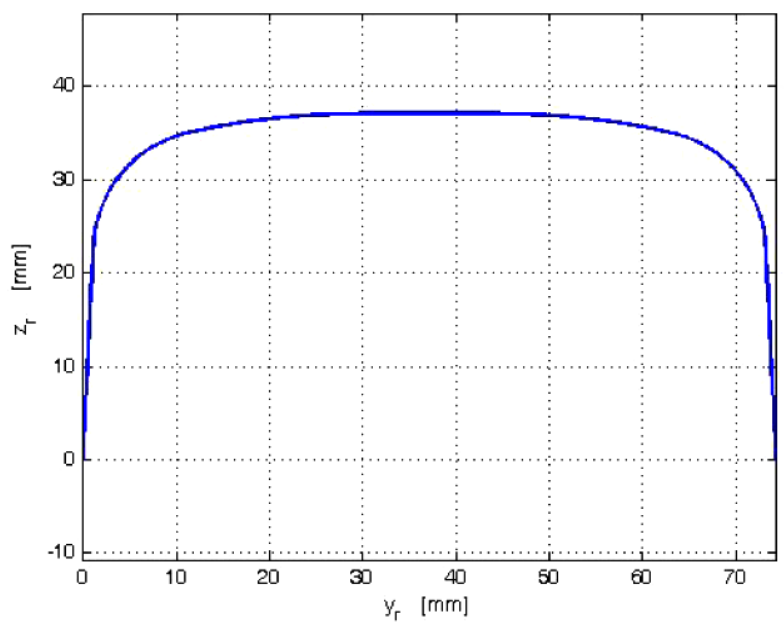

Fig. 9 Normal unitary vector on the wheel surface

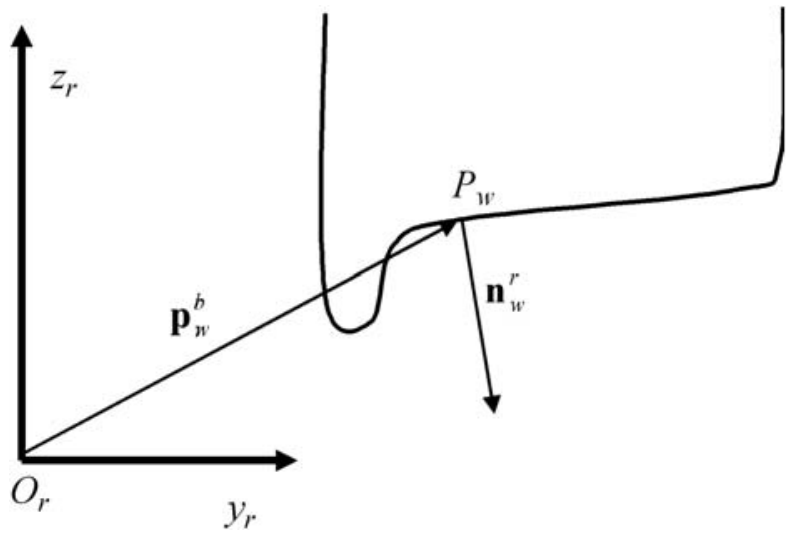

as follows:

$$
\begin{aligned}
\mathbf{n}_{w}^{w}\left(\mathbf{p}_{w}^{w}\right) & =-\left(\frac{\partial \mathbf{p}_{w}^{w}}{\partial x_{w}} \times \frac{\partial \mathbf{p}_{w}^{w}}{\partial y_{w}}\right) /\left\|\frac{\partial \mathbf{p}_{w}^{w}}{\partial x_{w}} \times \frac{\partial \mathbf{p}_{w}^{w}}{\partial y_{w}}\right\| \\
& =\left(\sqrt{\frac{r\left(y_{w}\right)^{2}\left(r^{\prime}\left(y_{w}\right)^{2}+1\right)}{r\left(y_{w}\right)^{2}-x_{w}^{2}}}\right)^{-1}\left[\begin{array}{c}
x_{w} / \sqrt{r\left(y_{w}\right)^{2}-x_{w}^{2}} \\
-r\left(y_{w}\right) r^{\prime}\left(y_{w}\right) / \sqrt{r\left(y_{w}\right)^{2}-x_{w}^{2}} \\
-1
\end{array}\right] .
\end{aligned}
$$

In this expression, $r^{\prime}\left(y_{w}\right)$ is the wheel profile derivative $r^{\prime}\left(y_{w}\right)=\frac{d r\left(y_{w}\right)}{d w}$. In the auxiliary reference system, the unitary vector normal to the wheel surface can be calculated as:

$$
\mathbf{n}_{w}^{r}\left(\mathbf{p}_{w}^{r}\right)=\left[\mathbf{R}_{2}\right] \mathbf{n}_{w}^{w}\left(\mathbf{p}_{w}^{w}\right)
$$

It is useful to remark that in this case $\sqrt{r\left(y_{w}\right)^{2}-x_{w}^{2}}$ is a real positive number since $r\left(y_{w}\right)^{2} \gg x_{w}^{2}$. 
Fig. 10 Normal unitary vector of the rail

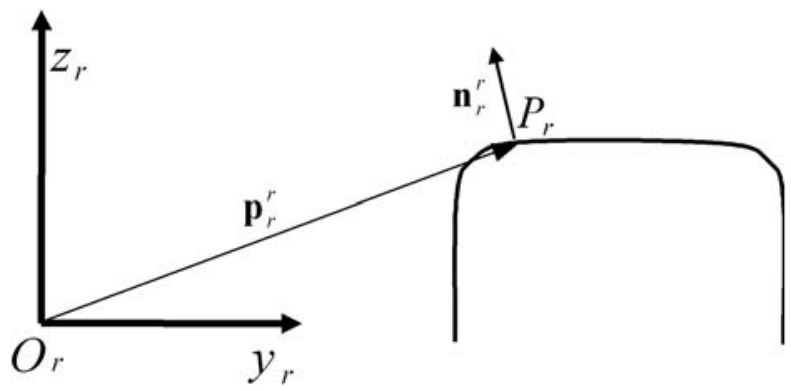

The unitary vector relative to the rail surface (Fig. 10), with respect to the auxiliary system is defined as:

$$
\begin{aligned}
\mathbf{n}_{r}^{r}\left(\mathbf{p}_{r}^{r}\right) & =\left(\frac{\partial \mathbf{p}_{r}^{r}}{\partial x_{r}} \times \frac{\partial \mathbf{p}_{r}^{r}}{\partial y_{r}}\right) /\left\|\frac{\partial \mathbf{p}_{r}^{r}}{\partial x_{r}} \times \frac{\partial \mathbf{p}_{r}^{r}}{\partial y_{r}}\right\| \\
& =\left(\sqrt{1+b^{\prime}\left(y_{r}\right)^{2}}\right)^{-1}\left[\begin{array}{c}
0 \\
-b^{\prime}\left(y_{r}\right) \\
1
\end{array}\right] .
\end{aligned}
$$

In this expression, $b^{\prime}\left(y_{r}\right)$ represents the rail profile derivative $b^{\prime}\left(y_{r}\right)=\frac{d b\left(y_{r}\right)}{d y_{r}}$.

\section{The DIST method}

As mentioned in the Introduction, the DIST method is based on the idea that in each contact point the distance between the wheel surface and the rail surface assumes a local maximum. The problem can be efficiently solved imposing the following conditions (Fig. 11) [14]:

- The normal unitary vector relative to the rail surface $\mathbf{n}_{r}^{r}\left(\mathbf{p}_{r}^{r}\right)$ has to be parallel to the wheel surface normal unitary vector $\mathbf{n}_{w}^{r}\left(\mathbf{p}_{w}^{r}\right)$;

$$
\mathbf{n}_{r}^{r} \times \mathbf{n}_{w}^{r}\left(\mathbf{p}_{w}^{r}\right)=\mathbf{n}_{r}^{r}\left(\mathbf{p}_{r}^{r}\right) \times\left[\mathbf{R}_{2}\right] \mathbf{n}_{w}^{w}\left(\mathbf{p}_{w}^{w}\right)=\mathbf{0} .
$$

- The rail surface normal unitary vector $\mathbf{n}_{r}^{r}\left(\mathbf{p}_{r}^{r}\right)$ has to be parallel to the vector representing the distance between the generic point of the wheel and of the rail $\mathbf{d}^{r}=\mathbf{p}_{w}^{r}-\mathbf{p}_{r}^{r}$

$$
\mathbf{n}_{r}^{r}\left(\mathbf{p}_{r}^{r}\right) \times \mathbf{d}^{r}=\mathbf{0} .
$$

The vector representing the distance between the generic point of the wheel and of the rail can be expressed as

$$
\mathbf{d}^{r}\left(x_{w}, y_{w}, x_{r}, y_{r}\right)=\mathbf{p}_{w}^{r}\left(x_{w}, y_{w}\right)-\mathbf{p}_{r}^{r}\left(x_{r}, y_{r}\right)=\mathbf{o}_{w}^{r}+\left[\mathbf{R}_{2}\right] \mathbf{p}_{w}^{w}\left(x_{w}, y_{w}\right)-\mathbf{p}_{r}^{r}\left(x_{r}, y_{r}\right)
$$

The vector $\mathbf{d}^{r}\left(x_{w}, y_{w}, x_{r}, y_{r}\right)$ depends on four parameters, namely the parameters used to identify a point on the rail and on the wheel surface, respectively. The conditions defined in (24) and (25) represent a system with six equations (since two vectorial constraints are 
Fig. 11 DIST method: vector representing the distance between the generic point of the wheel and of the rail

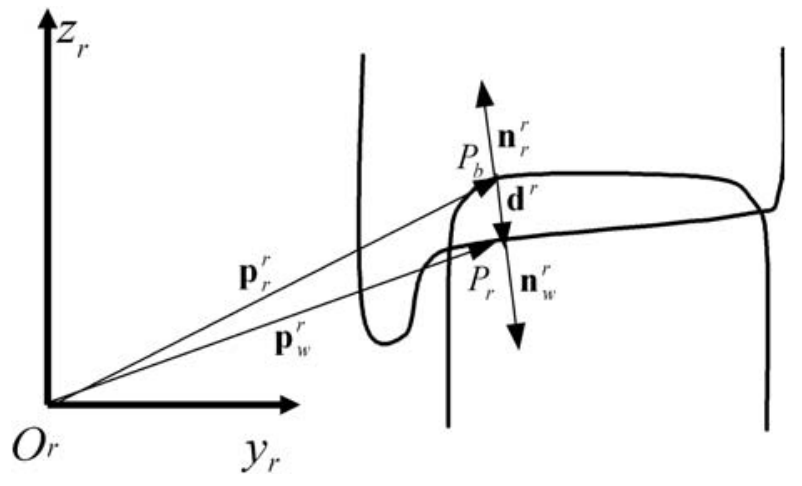

imposed) and four unknowns $\left(x_{w}, y_{w}, x_{r}, y_{r}\right)$, then only four of them are independent. Carrying out the calculations, it could be verified that the third component of both the vectorial equations (24) and (25) are proportional to the second one. Then only the first and second components of the equations can be considered for the solution of the problem.

The solutions of the system (24-25) are indicated with

$$
\left(x_{w i}^{C}, y_{w i}^{C}, x_{r i}^{C}, y_{w i}^{C}\right), \quad i=1,2, \ldots, n
$$

while

$$
\mathbf{p}_{w i}^{r, C}=\mathbf{p}_{w}^{r}\left(x_{w i}^{C}, y_{w i}^{C}\right), \quad \mathbf{p}_{r i}^{r, C}=\mathbf{p}_{r}^{r}\left(x_{r i}^{C}, y_{w i}^{C}\right), \quad i=1,2, \ldots, n
$$

are the corresponding contact points on the wheel and on the rail (Fig. 11).

The system solutions depends on the relative displacement between the wheelset and the rail, defined by the kinematic parameters $G_{y}, G_{z}, \alpha, \beta$. Because of the problem geometry, if the conditions $b\left(y_{r}\right)=b\left(-y_{r}\right)$ and $r\left(y_{w}\right)=r\left(-y_{w}\right)$ are satisfied (in other terms if the wheel and the rail profile are symmetric with respect to the $y_{w}$ and $y_{r}$ axis), the following symmetry conditions holds:

- If $\left(x_{w i}^{C}, y_{w i}^{C}, x_{r i}^{C}, y_{w i}^{C}\right)$ is a solution associated to the kinematic variables $\left(G_{y}, G_{z}, \alpha, \beta\right)$, then $\left(-x_{r i}^{C}, y_{w i}^{C},-x_{r i}^{C}, y_{r i}^{C}\right)$ will be a solution associated to the kinematic variables $\left(G_{y}, G_{z},-\alpha, \beta\right)$.

- If $\left(x_{w i}^{C}, y_{w i}^{C}, x_{r i}^{C}, y_{r i}^{C}\right)$ is a solution associated to the kinematic variables $\left(G_{y}, G_{z}, \alpha, \beta\right)$, then $\left(-x_{w i}^{C},-y_{w i}^{C},-x_{r i}^{C},-y_{r i}^{C}\right)$ will be a solution associated to the kinematic variables $\left(-G_{y}, G_{z}, \alpha,-\beta\right)$.

A generic solution of the system (24-25) can be considered an effective contact point only if the normal indentation between the surfaces $p_{n}$ is negative (according to our convention). Therefore, as regards the $i$-th solution, the following condition has to be verified:

$$
p_{n i}=\mathbf{d}_{i}^{r, C} \cdot \mathbf{n}_{r}^{r}\left(\mathbf{p}_{r i}^{r, C}\right) \leq 0,
$$

where $\mathbf{d}_{i}^{r, C}=\mathbf{p}_{w i}^{r, C}-\mathbf{p}_{r i}^{r, C}$. Otherwise, the solution must be rejected. 
The $i$-th solution has to pass also another test to be considered a contact point: the curvatures of the surfaces in the contact points have to satisfy some algebraic conditions so that the contact could be physically possible.

The normal principal curvatures $K_{1 w}\left(y_{w}\right)$ and $K_{2 w}\left(y_{w}\right)$ in a generic point of the wheel are defined as follows [14]:

$$
\begin{aligned}
K_{1 w}\left(y_{w}\right) & =\frac{1}{\left|r\left(y_{w}\right)\right| \sqrt{1+r^{\prime}\left(y_{w}\right)^{2}}}, \\
K_{2 w}\left(y_{w}\right) & =\frac{r^{\prime \prime}\left(y_{w}\right)}{\left(1+r^{\prime}\left(y_{w}\right)^{2}\right)^{3 / 2}},
\end{aligned}
$$

while the normal principal curvatures $K_{1 r}\left(y_{r}\right)$ and $K_{2 r}\left(y_{r}\right)$ in a generic point of the wheel are defined as:

$$
\begin{aligned}
& K_{1 r}\left(y_{r}\right)=0 \\
& K_{2 r}\left(y_{r}\right)=\frac{-b^{\prime \prime}\left(y_{r^{\prime}}\right)}{\left(1+b^{\prime}\left(y_{r^{\prime}}\right)^{2}\right)^{3 / 2}} .
\end{aligned}
$$

In these expressions, $r^{\prime \prime}\left(y_{w}\right)=\frac{d^{2} r\left(y_{w}\right)}{d y_{w}^{2}}$ and $b^{\prime \prime}\left(y_{r}\right)=\frac{d^{2} b\left(y_{r}\right)}{d y_{r}^{2}}$ represent the second derivative of the wheel and the rail profile, respectively. The curvatures are positive if the surfaces are convex (Fig. 12). Concerning the wheel principal directions of curvature the following relations hold:

$$
\begin{aligned}
& \mathbf{v}_{1 w}^{w}\left(x_{w}, y_{w}\right)=\frac{1}{\left|r\left(y_{w}\right)\right|}\left[\begin{array}{c}
\sqrt{r\left(y_{w}\right)^{2}-x_{w}^{2}} \\
0 \\
x_{w}
\end{array}\right], \\
& \mathbf{v}_{2 w}^{w}\left(x_{w}, y_{w}\right)=\frac{1}{\sqrt{1+r^{\prime}\left(y_{w}\right)^{2}}}\left[\begin{array}{c}
-\frac{r^{\prime}\left(y_{w}\right)}{\left|r\left(y_{w}\right)\right|} x_{w} \\
1 \\
\frac{r^{\prime}\left(y_{w}\right)}{\left|r\left(y_{w}\right)\right|} \sqrt{r\left(y_{w}\right)^{2}-x_{w}^{2}}
\end{array}\right] .
\end{aligned}
$$

Fig. 12 Signs of the normal principal curvatures

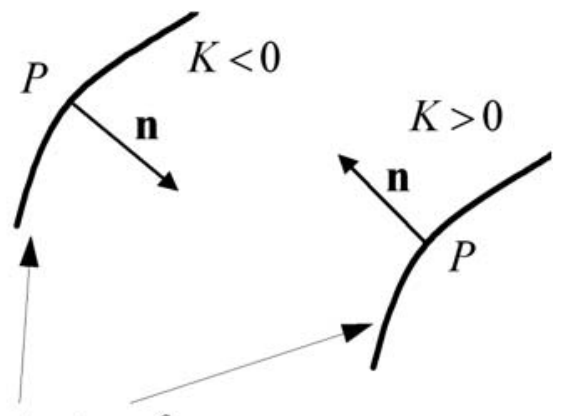

contact surfaces 
The rail principal directions of curvature are:

$$
\begin{aligned}
& \mathbf{v}_{1 r}^{r}\left(x_{r}, y_{r}\right)=\left[\begin{array}{l}
1 \\
0 \\
0
\end{array}\right], \\
& \mathbf{v}_{2 r}^{r}\left(x_{r}, y_{r}\right)=\frac{1}{\sqrt{1+b^{\prime}\left(y_{r}\right)^{2}}}\left[\begin{array}{c}
0 \\
1 \\
b^{\prime}\left(y_{r}\right)
\end{array}\right] .
\end{aligned}
$$

These relations can be used to calculate the normal curvatures $K_{1 r}\left(y_{r i}^{C}\right), K_{2 r}\left(y_{r i}^{C}\right), K_{1 w}\left(y_{w i}^{C}\right)$, and $K_{2 w}\left(y_{w i}^{C}\right)$ and the principal directions of curvature $\mathbf{v}_{1 r i}^{r, C}=\mathbf{v}_{1 r}^{r}\left(x_{r i}^{C}, y_{r i}^{C}\right), \mathbf{v}_{2 r i}^{r, C}=$ $\mathbf{v}_{2 r}\left(x_{r i}^{C}, y_{r i}^{C}\right), \mathbf{v}_{1 w i}^{w, C}=\mathbf{v}_{1 w}^{w}\left(x_{w i}^{C}, y_{w i}^{C}\right)$, and $\mathbf{v}_{2 w i}^{w, C}=\mathbf{v}_{2 w}\left(x_{w i}^{C}, y_{w i}^{C}\right)$ in the contact points.

Then the curvatures of the wheel and rail surfaces $k_{1 w i}^{C}, k_{2 w i}^{C}, k_{1 r i}^{C}, k_{2 r i}^{C}$ in the longitudinal and lateral rail direction can be calculated. The bundle of planes passing through the axis defined by the unitary vector $\mathbf{n}_{r}^{r}\left(\mathbf{p}_{r i}^{r, C}\right)$ defined on the point $\mathbf{p}_{r i}^{r, C}$ is considered (or equivalently the bundle defined by the axis passing through the unitary vector $\mathbf{n}_{w}^{r}\left(\mathbf{p}_{w i}^{r, C}\right)$ defined on the point $\mathbf{p}_{w i}^{w, C}$ ). Cutting the contact surfaces with the planes of the bundle containing the unitary vectors $\mathbf{v}_{1 r i}^{r, C}$ and $\mathbf{v}_{2 r i}^{r, C}$, by means of the Euler's formula, the following expressions for the curvature can be found [14]:

$$
\begin{aligned}
& k_{1 w i}^{C}=K_{1 w}\left(y_{w i}^{C}\right) \cos ^{2} \varphi_{i}^{C}+K_{2 w}\left(y_{w i}^{C}\right) \sin ^{2} \varphi_{i}^{C}, \\
& k_{2 w i}^{C}=K_{1 w}\left(y_{w i}^{C}\right) \cos ^{2} \psi_{i}^{C}+K_{2 w}\left(y_{w i}^{C}\right) \sin ^{2} \psi_{i}^{C}, \\
& k_{1 r i}^{C}=K_{1 r}\left(y_{w i}^{C}\right), \\
& k_{2 r i}^{C}=K_{2 r}\left(y_{r i}^{C}\right),
\end{aligned}
$$

where

$$
\begin{aligned}
\cos \varphi_{i}^{C} & =\mathbf{v}_{1 w i}^{w, C} \cdot \mathbf{v}_{1 r i}^{w, C}, \\
\cos \psi_{i}^{C} & =\mathbf{v}_{1 w i}^{w, C} \cdot \mathbf{v}_{2 r i}^{w, C}, \\
\mathbf{v}_{1 r i}^{w, C} & =\left[\mathbf{R}_{2}\right]^{T} \mathbf{v}_{1 r i}^{r, C}, \\
\mathbf{v}_{2 r i}^{w, C} & =\left[\mathbf{R}_{2}\right]^{T} \mathbf{v}_{2 r i}^{r, C} .
\end{aligned}
$$

Since $\psi_{i}^{C}=\varphi_{i}^{C}+\frac{\pi}{2}$, the following relation holds:

$$
k_{2 w i}^{C}=K_{1 w}\left(y_{w i}^{C}\right) \sin ^{2} \psi_{i}^{C}+K_{2 w}\left(y_{w i}^{C}\right) \cos ^{2} \psi_{i}^{C} .
$$

The generic solution $\left(x_{w i}^{C}, y_{w i}^{C}, x_{r i}^{C}, y_{r i}^{C}\right)$ with $i=1,2, \ldots, n$ of the system (24-25) can be considered an effective contact point only if the following conditions are verified:

$$
\begin{aligned}
& k_{1 r i}^{C}+k_{1 w i}^{C}>0, \\
& k_{2 r i}^{C}+k_{2 w i}^{C}>0 ;
\end{aligned}
$$

otherwise the contact is physically impossible. The situation is shown in Fig. 13 for the second direction, as it can be seen, in the point $P^{C, 2}$ the curvatures satisfy the inequality 
Fig. 13 Normal curvatures in the contact points

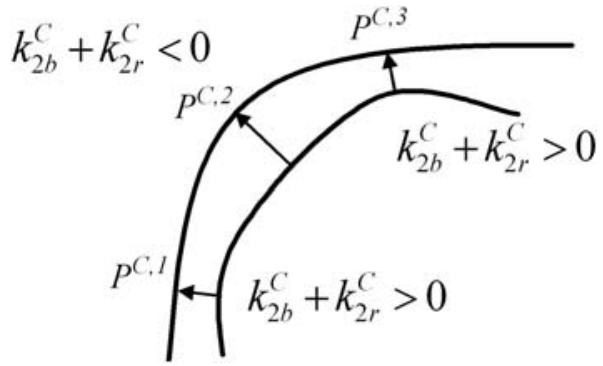

$k_{2 r i}^{C}+k_{2 w i}^{C}<0$, in other terms in this case the wheel curvature radius is larger than the rail one: even if the point $P^{C, 2}$ was a solution of the system (24-25) it could not be considered an effective contact point. Because of the geometry of the problem, the first of (36) conditions is always satisfied, and thus only the second has to be verified.

As hinted in the Introduction, the research of the distance stationary points is equivalent to solve an algebraic 4D-system defined by the first two components of the vectorial equations (24-25). However, the problem dimension can be analytically reduced from four to one. In other words, it is possible to express the variables $x_{w}, x_{r}, y_{r}$ as a function of $y_{w}$ in order to obtain a simple scalar equation in the unknown $y_{w}$.

From the second component of the vectorial equation defined in (24), the following expression can be found:

$$
r_{13} \sqrt{r\left(y_{w}\right)^{2}-x_{w}^{2}}=r_{11} x_{w}-r_{12} r\left(y_{w}\right) r^{\prime}\left(y_{w}\right) .
$$

In this expression, $r_{13}, r_{11}$, and $r_{12}$ are components of the matrix [ $\mathbf{R}_{2}$ ]. Indicating for brevity $A=r_{13}, B=r\left(y_{w}\right), C=r_{11}$, and $D=r_{12} r\left(y_{w}\right) r^{\prime}\left(y_{w}\right)$, it can be rewritten as:

$$
A \sqrt{B^{2}-x_{w}^{2}}=C x_{w}-D
$$

and, therefore, squaring both the members, it can be solved to obtain $x_{w}$ as a function of $y_{w}$ :

$$
x_{w 1,2}\left(y_{w}\right)=\frac{C D \pm \sqrt{C^{2} D^{2}-\left(C^{2}+A^{2}\right)\left(D^{2}-A^{2} B^{2}\right)}}{C^{2}+A^{2}} .
$$

As it can be seen, there are two possible values of $x_{w}$ for each value of $y_{w}$. From the first component of the vectorial equation defined in (24) the following expression can be found. The index $(1,2)$ indicates that the value has to be calculated for both the roots of (39)

$$
b^{\prime}\left(y_{r}\right)_{1,2}=\frac{r_{21} x_{w 1,2}\left(y_{w}\right)-r_{22} r\left(y_{w}\right) r^{\prime}\left(y_{w}\right)-r_{23} \sqrt{r\left(y_{w}\right)^{2}-x_{w 1,2}\left(y_{w}\right)^{2}}}{r_{32} r\left(y_{w}\right) r^{\prime}\left(y_{w}\right)+r_{33} \sqrt{r\left(y_{w}\right)^{2}-x_{w 1,2}\left(y_{w}\right)^{2}}} .
$$

The following condition:

$$
r_{32} r\left(y_{w}\right) r^{\prime}\left(y_{w}\right)+r_{33} \sqrt{r\left(y_{w}\right)^{2}-x_{w 1,2}\left(y_{w}\right)^{2}} \neq 0
$$

is always assured since

$$
r\left(y_{w}\right)^{2} \gg x_{w}^{2}, \quad r\left(y_{w}\right) \gg r^{\prime}\left(y_{w}\right) \quad \text { and } \quad r_{33} \gg r_{32} .
$$


Without loss of generality, the left and the right side of the track can be considered separately. In the first case, $y_{w} \in\left[\begin{array}{ll}700 & 790\end{array}\right] \mathrm{mm}$ and $y_{r} \in\left[\begin{array}{ll}720 & 780\end{array}\right] \mathrm{mm}$ and in the second $y_{w} \in\left[\begin{array}{ll}-790 & -700\end{array}\right] \mathrm{mm}$ and $y_{r} \in\left[\begin{array}{lll}-780 & -720\end{array}\right] \mathrm{mm}$. Under these assumptions, the function $b^{\prime}\left(y_{r}\right)$ is numerically invertible and the values of $y_{r 1,2}\left(y_{w}\right)$ can be calculated. Finally, from the second component of the vectorial equation defined in (25), the following relation can be written:

$$
x_{r 1,2}\left(y_{w}\right)=\mathbf{r}_{1} \cdot \mathbf{p}_{w}^{w}\left(x_{w 1,2}\left(y_{w}\right), y_{w}\right)=r_{11} x_{w 1,2}\left(y_{w}\right)+r_{12} y_{w}-r_{13} \sqrt{r\left(y_{w}\right)^{2}-x_{r 1,2}\left(y_{w}\right)^{2}}
$$

The variables $x_{w}, x_{r}, y_{r}$ have been expressed as a function of $y_{w}$ and can be introduced in the first component of the vectorial equation (25). Then the following relations can be found:

$$
\begin{aligned}
F_{1,2}\left(y_{w}\right)= & -b^{\prime}\left(y_{r 1,2}\left(y_{w}\right)\right)\left(G_{z}+\mathbf{r}_{3} \cdot \mathbf{p}_{w}^{w}\left(x_{w 1,2}\left(y_{w}\right), y_{w}\right)-b\left(y_{r 1,2}\left(y_{w}\right)\right)\right) \\
& -\left(G_{y}+\mathbf{r}_{2} \cdot \mathbf{p}_{w}^{w}\left(x_{w 1,2}\left(y_{w}\right), y_{w}\right)-y_{r 1,2}\left(y_{w}\right)\right) \\
= & -b^{\prime}\left(y_{r 1,2}\left(y_{w}\right)\right)\left(G_{z}+r_{32} y_{w}-r_{33} \sqrt{r\left(y_{w}\right)^{2}-x_{w 1,2}\left(y_{w}\right)^{2}}-b\left(y_{r 1,2}\left(y_{w}\right)\right)\right) \\
& -\left(G_{y}+r_{21} x_{w 1,2}\left(y_{w}\right)+r_{22} y_{w}-r_{23} \sqrt{r\left(y_{w}\right)^{2}-x_{w 1,2}\left(y_{w}\right)^{2}}-y_{r 1,2}\left(y_{w}\right)\right)=0 .
\end{aligned}
$$

It is a simple scalar equation in the variable $y_{w}$ (where $y_{w} \in\left[\begin{array}{lll}700 & 790\end{array}\right] \mathrm{mm}$ for the left side and $y_{w} \in\left[\begin{array}{ll}-790-700\end{array}\right] \mathrm{mm}$ for the right side) and can be solved numerically. Also, in this case, the index $(1,2)$ indicates that the equation has to be solved for both the roots of (39).

For simplicity, the solutions $y_{w i}^{C}$ (with $i=1,2, \ldots, n$ ) of (43) are split in two groups: $y_{w 1 j}^{C}$ (with $\left.j=1,2, \ldots, n_{1}\right)$ indicates the roots of $F_{1}\left(y_{w}\right)=0$ (obtained with the first root of (39)) and $y_{w 2 k}^{C}$ (with $\left.k=1,2, \ldots, n_{2}\right)$ those of $F_{2}\left(y_{w}\right)=0$, where $n=n_{1}+n_{2}$. Through (39), (40), and (42), the values of the variables $x_{w}, x_{r}, y_{r}$ corresponding to $y_{w 1 j}^{C}$ and $y_{w 2 k}^{C}$ can be calculated:

$$
\begin{aligned}
& x_{w 1 j}^{C}=x_{w 1}\left(y_{w 1 j}^{C}\right), \quad x_{r 1 j}^{C}=x_{r 1}\left(y_{w 1 j}^{C}\right), \quad y_{r 1 j}^{C}=y_{r 1}\left(y_{w 1 j}^{C}\right), \quad j=1,2, \ldots, n_{1}, \\
& x_{w 2 k}^{C}=x_{w 2}\left(y_{w 2 k}^{C}\right), \quad x_{r 2 k}^{C}=x_{r 2}\left(y_{w 2 k}^{C}\right), \quad y_{r 2 k}^{C}=y_{r 2}\left(y_{w 2 k}^{C}\right), \quad k=1,2, \ldots, n_{2}
\end{aligned}
$$

and, therefore, by means of (15), (16), and (19), the positions of the contact points on the wheel and on the rail are calculated:

$$
\begin{aligned}
& \mathbf{p}_{w 1 j}^{r, C}=\mathbf{p}_{w}^{r}\left(x_{w 1 j}^{C}, y_{w 1 j}^{C}\right), \quad \mathbf{p}_{r 1 j}^{r, C}=\mathbf{p}_{r}^{r}\left(x_{r 1 j}^{C}, y_{r 1 j}^{C}\right), \quad j=1,2, \ldots, n_{1}, \\
& \mathbf{p}_{w 2 k}^{r, C}=\mathbf{p}_{w}^{r}\left(x_{w 2 k}^{C}, y_{w 2 k}^{C}\right), \quad \mathbf{p}_{r 2 k}^{r, C}=\mathbf{p}_{r}^{r}\left(x_{r 2 k}^{C}, y_{r 2 k}^{C}\right), \quad k=1,2, \ldots, n_{2} .
\end{aligned}
$$

Not all the solutions $\left(x_{w 1 j}^{C}, y_{w 1 j}^{C}, x_{r 1 j}^{C}, y_{r 1 j}^{C}\right)$ (with $\left.j=1,2, \ldots, n_{1}\right)$ and $\left(x_{w 2 k}^{C}, y_{w 2 k}^{C}\right.$, $x_{r 2 k}^{C}, y_{r 2 k}^{C}$ ) (with $k=1,2, \ldots, n_{2}$ ) obtained finding the roots of (43) can be accepted, since (37)-(43) contain irrational terms. Consequently, the following conditions have to be veri- 
fied for every $j$ and $k$ :

- $x_{w 1 j}^{C}$ and $x_{w 2 k}^{C}$ (calculated by (39) as a function of $y_{w 1 j}^{C}$ and $y_{w 2 k}^{C}$ ) have to be real numbers.

- The terms $\sqrt{r\left(y_{w 1 j}^{C}\right)^{2}-\left(x_{w 1 j}^{C}\right)^{2}}$ and $\sqrt{r\left(y_{w 2 k}^{C}\right)^{2}-\left(x_{w 2 k}^{C}\right)^{2}}$ appearing in (43) have to be real.

- $\left(x_{w 1 j}^{C}, y_{w 1 j}^{C}\right)$ and $\left(x_{w 2 k}^{C}, y_{w 2 k}^{C}\right)$ have to be effective solutions of (37).

If one of these conditions is not verified, the solution has to be rejected. Moreover, concerning eventual multiple solutions, if $m$ is the multiplicity of the solution, only one of these can be considered because the others $m-1$ have not physical meaning. The multiplicity of the solution has to be evaluated considering all the solutions in both the sets $\left(x_{w 1 j}^{C}, y_{w 1 j}^{C}, x_{r 1 j}^{C}, y_{r 1 j}^{C}\right)$ (with $\left.j=1,2, \ldots, n_{1}\right)$ and $\left(x_{w 2 k}^{C}, y_{w 2 k}^{C}, x_{r 2 k}^{C}, y_{r 2 k}^{C}\right)$ (with $k=$ $\left.1,2, \ldots, n_{2}\right)$.

For each wheel/rail configuration, the DIST method requires therefore the following steps:

1. Determination of the solutions $\left(x_{w i}^{C}, y_{w i}^{C}, x_{r i}^{C}, y_{r i}^{C}\right)$ (with $\left.i=1,2, \ldots, n\right)$ of the system (24-25)

2. Research and elimination of the multiple solutions

3. Check of the analytic conditions

4. Check of the condition on the curvatures

5. Check of the condition on the normal indentation.

These controls allow to verify that the solutions are physically realistic, in the analytic development no simplifications and approximations were assumed then no solution of the problem should be excluded.

\section{The DIFF method}

The DIFF method has been developed in order to simplify the method described in the preceding section and then to further improve its efficiency. The DIFF method is based on the idea that the contact points minimize the difference between the wheel surface and the rail surface in the direction identified by the unitary vector $\mathbf{k}_{r}$ :

$$
D\left(x_{w}, y_{w}\right)=\left(\mathbf{p}_{w}^{r}\left(x_{w}, y_{w}\right)-\mathbf{p}_{r}^{r}\left(x_{w}, y_{w}\right)\right) \cdot \mathbf{k}_{r},
$$

where $\mathbf{p}_{w}^{r}$ is defined according to (15), while $\mathbf{p}_{r}^{r}\left(x_{w}, y_{w}\right)$ is defined as follows:

$$
\mathbf{p}_{r}^{r}\left(x_{w}, y_{w}\right)=\left[\begin{array}{c}
x_{w}^{r}\left(x_{w}, y_{w}\right) \\
y_{w}^{r}\left(x_{w}, y_{w}\right) \\
b\left(y_{w}^{r}\left(x_{w}, y_{w}\right)\right)
\end{array}\right] .
$$

In other terms, the point $\mathbf{p}_{r}^{r}\left(x_{w}, y_{w}\right)$ is evaluated as the intersection between the rail surface and a line parallel to the axis $z_{r}$ passing through the point $\mathbf{p}_{w}^{r}$ on the wheel surface (Fig. 14). For each wheel/rail relative configuration, the difference $D\left(x_{w}, y_{w}\right)$ is then a function depending on two variables (in other terms it is a two dimensional surface).

The method is based on an approximation that could be a priori unacceptable, it is approximately true when considering wheel-rail tread contact, but it may be wrong when considering wheel-rail flange contact. However, it allows to simplify the problem and to obtain 
Fig. 14 Minimization of the difference: definition of the difference function

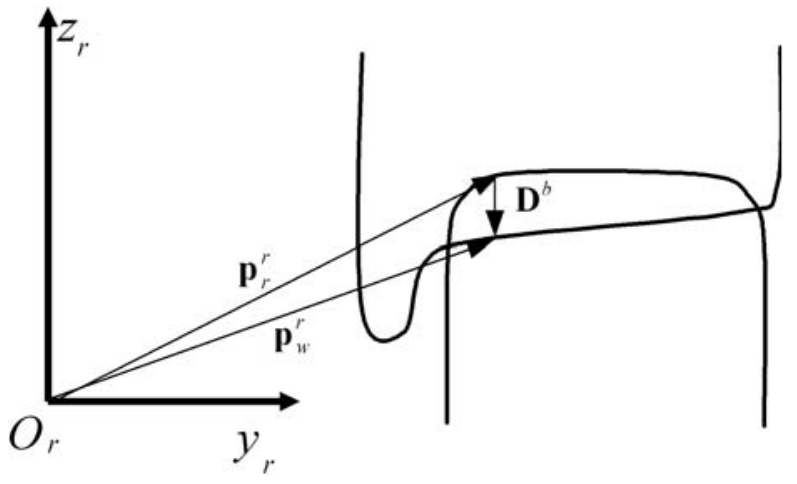

a procedure with an higher computational efficiency that could be implemented directly in the multibody code. In order to verify the reliability of the proposed procedure, its results have been compared with those obtained with the DIST method described above. The results of the tests are summarized in Sect. 5.

The contact points between the surfaces can be approximated with the minima of the difference function. In some preceding works $[10,11]$, the authors presented some procedures to find numerically the minima, based on iterative methods (namely Simplex method and Compass search $[12,13])$. The efficiency of these methods was not sufficiently high to allow an on-line implementation of the procedure.

The problem could be solved analytically imposing the following conditions on the partial derivatives of the function $D\left(x_{w}, y_{w}\right)$ [14]:

$$
\frac{\partial D\left(x_{w}, y_{w}\right)}{\partial x_{w}}=0, \quad \frac{\partial D\left(x_{w}, y_{w}\right)}{\partial y_{w}}=0 .
$$

Also, in this case, $\left(x_{w i}^{C}, y_{w i}^{C}\right)$ with $i=1,2, \ldots, n$ indicate the solutions of the system (48), and $\mathbf{p}_{w i}^{r, C}=\mathbf{p}_{w}^{r}\left(x_{w i}^{C}, y_{w i}^{C}\right), \mathbf{p}_{r i}^{r, C}=\mathbf{p}_{r}^{r}\left(x_{w i}^{C}, y_{w i}^{C}\right)$ with $i=1,2, \ldots, n$ the corresponding contact points on the wheel and on the rail (for each wheelset/rail configuration, $n$ is the total number of contact points).

Because of the problem geometry, if $b\left(y_{r}\right)=b\left(-y_{r}\right)$ and $r\left(y_{w}\right)=r\left(-y_{w}\right)$, the same symmetry conditions described in the preceding section are verified.

To be considered possible contact points, the solutions of the system (48) have to be effectively a minimum of the surface (46), then the Hessian matrix $H_{D}\left(x_{w}, y_{w}\right)$ of $D\left(x_{w}, y_{w}\right)$, defined as [14]:

$$
H_{D}\left(x_{w}, y_{w}\right)=\left(\begin{array}{cc}
\frac{\partial^{2} D}{\partial x_{w}^{2}} & \frac{\partial^{2} D}{\partial x_{w} \partial y_{w}} \\
\frac{\partial^{2} D}{\partial x_{w} \partial y_{w}} & \frac{\partial^{2} D}{\partial y_{w}^{2}}
\end{array}\right)
$$

has to be positive defined in the points $\left(x_{w i}^{C}, y_{w i}^{C}\right)$ with $i=1,2, \ldots, n$. Since $D\left(x_{w}, y_{w}\right)$ : $\mathbb{R}^{2} \rightarrow \mathbb{R}$, this is equivalent to

$$
\frac{\partial^{2} D}{\partial x_{w}^{2}}\left(x_{w i}^{C}, y_{w i}^{C}\right)>0, \quad \operatorname{det} H_{D}\left(x_{w i}^{C}, y_{w i}^{C}\right)>0
$$

with $i=1,2, \ldots, n$.

The generic solution of (48) can be considered an effective contact point only if the normal indentation between the surfaces $p_{n}$ is negative. In this case, the normal indentation 
Fig. 15 Minimization of the difference: definition of the surface indentation

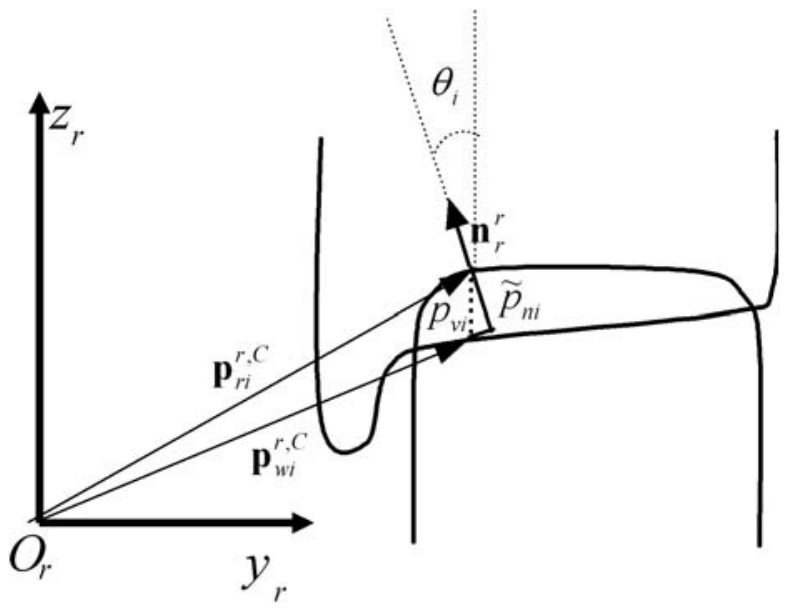

$p_{n}$ cannot be calculated directly from the values of $x_{w}, y_{w}$ because the normal to the contact surfaces in the contact point is not unique with this approach. For this reason, it was approximated with the value $\tilde{p}_{n i}$ calculated as follows (Fig. 15):

$$
\tilde{p}_{n i}=\mathbf{D}_{i}^{r, C} \cdot \mathbf{n}_{r}^{r}\left(\mathbf{p}_{r i}^{r, C}\right)=p_{v i} \cos \vartheta_{i}
$$

In this expression, $\mathbf{D}_{i}^{r, C}$ is the vector representing the difference between the surfaces, defined as:

$$
\mathbf{D}_{i}^{r, C}=\mathbf{p}_{w i}^{r, C}-\mathbf{p}_{w i}^{r, C},
$$

$p_{v i}$ represents the indentation evaluated in the $z_{r}$ direction, defined as:

$$
p_{v i}=\mathbf{D}_{i}^{r, C} \cdot \mathbf{k}_{r},
$$

and $\vartheta_{i}$ is the angle between the $z_{r}$ direction and the unitary vector normal to the rail surface:

$$
\cos \vartheta_{i}=\mathbf{n}_{r}^{r}\left(\mathbf{p}_{r i}^{r, C}\right) \cdot \mathbf{k}_{r}
$$

Therefore, for each solution of (48), the following condition has to be verified:

$$
\tilde{p}_{n i}=\leq 0 .
$$

The minimization of the difference is equivalent then to solve an algebraic 2D-system (48). However, also in this case, the problem dimension can be analytically reduced, since it is possible to express the variable $x_{w}$ as a function of $y_{w}$ in order to obtain a simple scalar equation in the variable $y_{w}$. Introducing the definitions of $\mathbf{p}_{w}^{r}\left(x_{w}, y_{w}\right)$ and $\mathbf{p}_{r}^{r}\left(x_{w}, y_{w}\right)$ into (46), the following expression can be found:

$$
\begin{aligned}
D\left(x_{w}, y_{w}\right) & =\left(\mathbf{p}_{w}^{r}\left(x_{w}, y_{w}\right)-\mathbf{p}_{r}^{r}\left(x_{w}, y_{w}\right)\right) \cdot \mathbf{k}_{r}=z_{w}^{r}\left(x_{w}, y_{w}\right)-b\left(y_{w}^{r}\left(x_{w}, y_{w}\right)\right) \\
& =G_{z}+\mathbf{r}_{3} \cdot \mathbf{p}_{r}^{r}\left(x_{w}, y_{w}\right)-b\left(G_{y}+\mathbf{r}_{2} \cdot \mathbf{p}_{w}^{w}\left(x_{w}, y_{w}\right)\right) .
\end{aligned}
$$


The partial derivatives of the function (48) are:

$$
\begin{aligned}
& \frac{\partial D}{\partial x_{w}}\left(x_{w}, y_{w}\right)=\mathbf{r}_{3} \cdot \frac{\partial \mathbf{p}_{w}^{w}}{\partial x_{w}}\left(x_{w}, y_{w}\right)-b^{\prime}\left(G_{y}+\mathbf{r}_{2} \cdot \mathbf{p}_{w}^{w}\left(x_{w}, y_{w}\right)\right) \mathbf{r}_{2} \cdot \frac{\partial \mathbf{p}_{w}^{w}}{\partial x_{w}}\left(x_{w}, y_{w}\right)=0, \\
& \frac{\partial D}{\partial y_{w}}\left(x_{w}, y_{w}\right)=\mathbf{r}_{3} \cdot \frac{\partial \mathbf{p}_{w}^{w}}{\partial y_{w}}\left(x_{w}, y_{w}\right)-b^{\prime}\left(G_{y}+\mathbf{r}_{2} \cdot \mathbf{p}_{w}^{w}\left(x_{w}, y_{w}\right)\right) \mathbf{r}_{2} \cdot \frac{\partial \mathbf{p}_{w}^{w}}{\partial y_{w}}\left(x_{w}, y_{w}\right)=0 .
\end{aligned}
$$

The last term of (55) and (56) can be rewritten as follows:

$$
\mathbf{r}_{2} \cdot \frac{\partial \mathbf{p}_{w}^{w}}{\partial y_{w}}\left(x_{w}, y_{w}\right)=\left(r_{22}-r_{23} \frac{r\left(y_{w}\right) r^{\prime}\left(y_{w}\right)}{\sqrt{r\left(y_{w}\right)^{2}-x_{w}^{2}}}\right),
$$

and its value is different from zero, since $r\left(y_{w}\right)^{2} \gg x_{w}^{2}$ and $r_{22} \gg r_{23} r^{\prime}\left(y_{w}\right)$. The term $b^{\prime}\left(G_{y}+\mathbf{r}_{2} \cdot \mathbf{p}_{w}^{w}\left(x_{w}, y_{w}\right)\right)$ can be obtained from (56) and can be introduced in (55), in order to achieve:

$$
\left(\mathbf{r}_{3} \cdot \frac{\partial \mathbf{p}_{w}^{w}}{\partial x_{w}}\left(x_{w}, y_{w}\right)\right)\left(\mathbf{r}_{2} \cdot \frac{\partial \mathbf{p}_{w}^{w}}{\partial y_{w}}\left(x_{w}, y_{w}\right)\right)=\left(\mathbf{r}_{2} \cdot \frac{\partial \mathbf{p}_{w}^{w}}{\partial x_{w}}\left(x_{w}, y_{w}\right)\right)\left(\mathbf{r}_{3} \cdot \frac{\partial \mathbf{p}_{w}^{w}}{\partial y_{w}}\left(x_{w}, y_{w}\right)\right)
$$

and, carrying out the calculations:

$$
r_{21} r_{32} \sqrt{r\left(y_{w}\right)^{2}-x_{w}^{2}}=\left(r_{22} r_{33}-r_{23} r_{32}\right) x_{w}+r_{21} r_{33} r\left(y_{w}\right) r^{\prime}\left(y_{w}\right) .
$$

Setting for brevity $A_{1}=r_{21} r_{32}, B_{1}=r\left(y_{w}\right), C_{1}=r_{22} r_{33}-r_{23} r_{32}$, and $D_{1}=r_{21} r_{33} r\left(y_{w}\right) \times$ $r^{\prime}\left(y_{w}\right),(59)$ can be rewritten as:

$$
A_{1} \sqrt{B_{1}^{2}-x_{w}^{2}}=C_{1} x_{w}+D_{1}
$$

and, therefore, squaring both the members, the term $x_{w}$ can be calculated:

$$
x_{w 1,2}\left(y_{w}\right)=\frac{-C_{1} D_{1} \pm \sqrt{C_{1}^{2} D_{1}^{2}-\left(C_{1}^{2}+A_{1}^{2}\right)\left(D_{1}^{2}-A_{1}^{2} B_{1}^{2}\right)}}{C_{1}^{2}+A_{1}^{2}} .
$$

The variable $x_{w}$ is then expressed as a function of $y_{w}$ and as it can be seen, there are again two possible values of $x_{w}$ for each value of $y_{w}$. Finally, we can introduce the relation (61) in the second member of (55) obtaining:

$$
\begin{aligned}
F_{1,2}\left(y_{w}\right)= & \frac{\partial D\left(x_{w 1,2}\left(y_{w}\right), y_{w}\right)}{\partial y_{w}} \\
= & \mathbf{r}_{3} \cdot \frac{\partial \mathbf{p}_{w}^{w}\left(x_{w 1,2}\left(y_{w}\right), y_{w}\right)}{\partial y_{w}}-b^{\prime}\left(G_{y}+\mathbf{r}_{2} \cdot \mathbf{p}_{w}^{w}\left(x_{w 1,2}\left(y_{w}\right), y_{w}\right)\right) \mathbf{r}_{2} \cdot \frac{\partial \mathbf{p}_{w}^{w}\left(x_{w 1,2}\left(y_{w}\right), y_{w}\right)}{\partial y_{w}} \\
= & r_{32} y_{w}-r_{33} \frac{r\left(y_{w}\right) r^{\prime}\left(y_{w}\right)}{\sqrt{r\left(y_{w}\right)^{2}-x_{r 1,2}\left(y_{w}\right)^{2}}} \\
& -b^{\prime}\left(G_{y}+r_{21} x_{w 1,2}\left(y_{w}\right)+r_{22} y_{w}-r_{23} \sqrt{\left.r\left(y_{w}\right)^{2}-x_{r 1,2}\left(y_{w}\right)^{2}\right)}\right. \\
& \times\left(r_{22}-r_{23} \frac{r\left(y_{w}\right) r^{\prime}\left(y_{w}\right)}{\sqrt{r\left(y_{w}\right)^{2}-x_{w 1,2}\left(y_{w}\right)^{2}}}\right)=0
\end{aligned}
$$


The problem is reduced to a simple scalar equation in the variable $y_{w}$ (where $y_{w} \in$ [700 790] $\mathrm{mm}$ for the left side and $y_{w} \in\left[\begin{array}{lll}-790 & -700\end{array} \mathrm{~mm}\right.$ for the right side) that can be solved numerically [12]. Also, in this case, the index $(1,2)$ indicates that the equation has to be solved for both the roots of (61).

As usual for simplicity, the solutions $y_{w i}^{C}$ (with $\left.i=1,2, \ldots, n\right)$ of (62) are split in two groups: $y_{w 1 j}^{C}$ (with $\left.j=1,2, \ldots, n_{1}\right)$ indicate the roots of $F_{1}\left(y_{w}\right)=0$ (obtained using the first root of (61)) and $y_{w 2 k}^{C}$ (with $\left.k=1,2, \ldots, n_{2}\right)$ those of $F_{2}\left(y_{w}\right)=0$ where $n=$ $n_{1}+n_{2}$ (obtained using the second root of (61)). Through (61), the values of the variable $x_{w}$ corresponding to $y_{w 1 j}^{C}$ and $y_{w 2 k}^{C}$ can be evaluated:

$$
\begin{array}{ll}
x_{w 1 j}^{C}=x_{w 1}\left(y_{r 1 j}^{C}\right), & j=1,2, \ldots, n_{1}, \\
x_{w 2 k}^{C}=x_{w 2}\left(y_{r 2 k}^{C}\right), & k=1,2, \ldots, n_{2}
\end{array}
$$

and, therefore, by means of (15), (16), and (47), the positions of the contact points on the wheel and on the rail surfaces can be calculated:

$$
\begin{aligned}
& \mathbf{p}_{w 1 j}^{r, C}=\mathbf{p}_{w}^{r}\left(x_{w 1 j}^{C}, y_{w 1 j}^{C}\right), \quad \mathbf{p}_{r 1 j}^{r, C}=\mathbf{p}_{r}^{r}\left(x_{w 1 j}^{C}, y_{w 1 j}^{C}\right), \quad j=1,2, \ldots, n_{1}, \\
& \mathbf{p}_{w 2 k}^{r, C}=\mathbf{p}_{w}^{r}\left(x_{w 2 k}^{C}, y_{w 2 k}^{C}\right), \quad \mathbf{p}_{r 2 k}^{r, C}=\mathbf{p}_{r}^{r}\left(x_{w 2 k}^{C}, y_{w 2 k}^{C}\right), \quad k=1,2, \ldots, n_{2} .
\end{aligned}
$$

As in the case of the DIST method, not all the solutions $\left(x_{w 1 j}^{C}, y_{w 1 j}^{C}\right)$ (with $\left.j=1,2, \ldots, n_{1}\right)$ and $\left(x_{w 2 k}^{C}, y_{w 2 k}^{C}\right)$ (with $\left.k=1,2, \ldots, n_{2}\right)$ of (62) can be accepted. Equation (59) contains irrational terms, and consequently the following analytic conditions have to be verified for every $j$ and $k$ :

1. $x_{w 1 j}^{C}$ and $x_{w 2 k}^{C}$ calculated by means of (61) have to be real.

2. The terms $\sqrt{r\left(y_{w 1 j}^{C}\right)^{2}-\left(x_{w 1 j}^{C}\right)^{2}}$ and $\sqrt{r\left(y_{w 2 k}^{C}\right)^{2}-\left(x_{w 2 k}^{C}\right)^{2}}$ appearing in (62) have to be real.

3. $\left(x_{w 1 j}^{C}, y_{w 1 j}^{C}\right)$ and $\left(x_{w 2 k}^{C}, y_{w 2 k}^{C}\right)$ have to be effective solutions of (59).

Moreover, as regards eventual multiple solutions, the same considerations explained for the DIST method are necessary.

The DIFF method requires, therefore, the following steps:

1. Determination of the solutions $\left(x_{w i}^{C}, y_{w i}^{C}\right)$ (with $\left.i=1,2, \ldots, n\right)$ of (48)

2. Research and elimination of the multiple solutions

3. Check of the analytic conditions

4. Check of the minimum condition and

5. Check of the condition on the normal indentation.

\section{Numerical results}

In this section, the numerical results of the proposed procedures will be illustrated in order to evaluate the performances of the new procedures for the detection of the contact points. In the first part, some simulations of a standard railway vehicle dynamics are described. The simulations have been carried out by means of a multibody 3D model within which the described methods for the evaluation of the contact point have been implemented. In the second part, the performances of the proposed procedures are be compared with those of the methods present in literature. Particular attention will be dedicated to the computation burden and to the precision of the methods [10-13]. 


\subsection{Dynamic simulations}

The railway vehicle chosen for the dynamic simulations is the Manchester Wagon whose physical and geometric characteristics are easily available in the literature [16]. The multibody $3 \mathrm{D}$ model of this vehicle has been studied and validated in different conditions. Consequently, the model can be considered a reliable benchmark for the evaluation of the performances of the previously presented procedures [10, 11]. The multibody 3D model of the Manchester Wagon, implemented in the Matlab computation environment, is schematically shown in Fig. 16.

The vehicle is composed of the following parts:

1. The car body (Fig. 16)

2. Two bogies (Figs. 17a and b)

3. Four axles (Fig. 17c)

4. Primary and secondary suspensions modeled by three-dimensional nonlinear force elements like bushings, dampers, and bumpstops (Figs. 17b and c).

The contact point locations are calculated with the procedures described in the preceding section. The indentation value is used to calculate the normal component of the contact force, $N$, according to the elasto-viscous approach as sum of a term that depends on the normal indentation between the bodies (the elastic component), and a term proportional to the surface relative velocities in the contact point (the viscous term):

$$
N=\left(-k_{h}|p|^{\gamma}+k_{v}|v| \frac{\operatorname{sign}(v)-1}{2}\right) \frac{\operatorname{sign}(p)-1}{2} .
$$

In this expression:

- $k_{h}$ is a constant that is calculated according to Hertz theory as a function of the surface geometries and of the material characteristics [10,11].

- $p$ is the indentation in the contact point, defined as described in (29) if the DIST method is used and as described in (51) if the DIFF method is used.

- $\gamma$ is a constant derived from Hertz theory, in this case $\gamma=\frac{3}{2}$ [17].

- $k_{v}$ is a constant depending on material properties, its value has been chosen on the basis of data available in the literature $\left(k_{v}=10^{4} \mathrm{Ns} / \mathrm{m}\right)[2,11]$.

- $v$ is the indentation velocity.

The indentation velocity $v$ is defined on the basis of the relative speed between the contact bodies in the contact point $\mathbf{v}_{c}$ :

$$
v=\mathbf{v}_{c}^{r} \cdot \mathbf{n}_{r}^{r} .
$$

Fig. 16 Multibody 3D model of the Manchester Wagon

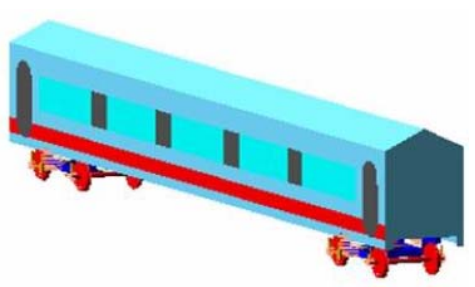




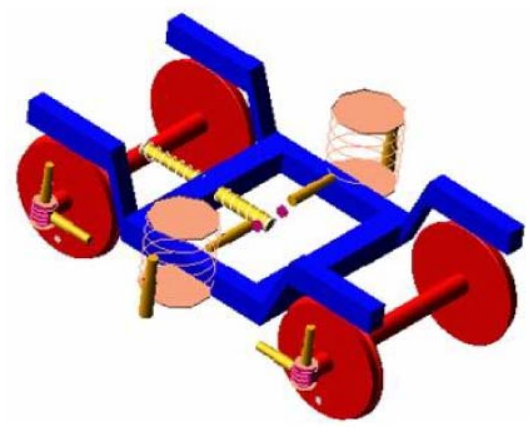

(a)

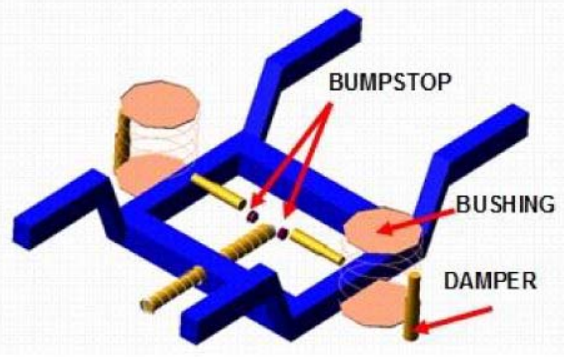

(b)

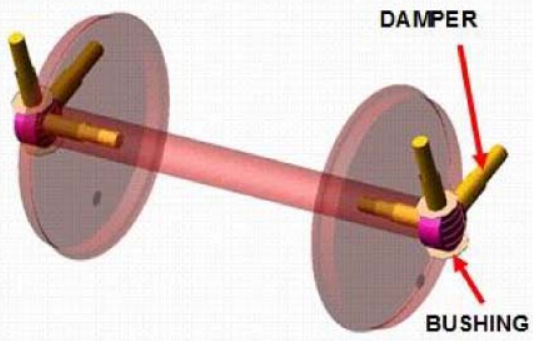

(c)

Fig. 17 Bogie, suspensions, and axle of the Manchester Wagon, (a) vehicle bogie, (b) secondary suspensions, and (c) primary suspensions and axle

The relative speed on the contact points can be calculated from the kinematics of the wheelset:

$$
\mathbf{v}_{c}^{r}=\mathbf{v}_{o w}^{r}+\boldsymbol{\Omega}_{w}^{r} \times\left(\mathbf{p}_{w}^{r C}-\mathbf{o}_{w}^{r}\right),
$$

- $\mathbf{v}_{c}^{r}$ is the wheel speed in the contact point expressed with respect to the railway auxiliary reference system.

- $\mathbf{v}_{o w}^{r}$ is the wheelset center of mass velocity.

- $\boldsymbol{\Omega}_{w}^{r}$ is the wheelset angular speed.

- $\mathbf{p}_{w}^{r C}$ is the contact point location expressed with respect to the auxiliary reference system.

- $\mathbf{o}_{w}^{r}$ is the wheelset center of mass location expressed with respect to the auxiliary reference system.

The magnitude of the tangential component of the contact forces is calculated on the basis of Kalker and Hertz theory $[9,17,18]$. The Hertz's theory is used to define the contact area dimensions and shape that depend on the normal force magnitude, the material properties, and the local profile geometry. The Kalker linear theory results are used to define the components of the creep forces as follows:

$$
\begin{aligned}
T_{x} & =-f_{11} \xi, \\
T_{y} & =-f_{22} \eta-f_{23} \phi, \\
M_{s p} & =f_{23} \eta-f_{33} \phi,
\end{aligned}
$$


where $T_{x}$ and $T_{y}$ represent respectively the longitudinal and lateral component of the contact force, $M_{s p}$ is the spin moment. The coefficients $f_{11}, f_{22}, f_{33}, f_{23}$ are the linear creep coefficients, depending on the contact ellipse semiaxis and on the material properties, their values are tabulated and can be found, for example, in [17]. The coefficients $\xi, \eta$, and $\phi$ represent respectively the longitudinal, lateral, and spin creepage components and are defined as follows [9]:

$$
\begin{gathered}
\xi=\frac{\mathbf{v}_{c}^{r} \cdot \mathbf{i}_{r}}{v_{o w}}, \\
\eta=\frac{\mathbf{v}_{c}^{r} \cdot \mathbf{t}_{r}}{v_{o w}}, \\
\phi=\frac{\boldsymbol{\Omega}_{w}^{r} \cdot \mathbf{n}_{r}}{v_{o w}} .
\end{gathered}
$$

In the above expressions, $\mathbf{v}_{c}^{r}$ is the wheel speed in the contact point calculated as shown in (67), $\mathbf{i}_{r}$ is the unitary vector that identify the rail longitudinal direction, $\mathbf{t}_{r}$ is the unitary vector tangent to the contact surface and orthogonal to $\mathbf{i}_{r}$ (it identifies the lateral direction), $\mathbf{n}_{r}$ is the unitary vector normal to the contact surfaces in the contact point, $v_{o w}$ is the magnitude of the wheelset center of mass velocity.

The unlimited resultant creep force obtained from the linear model is:

$$
T_{r}=\sqrt{T_{x}^{2}+T_{y}^{2}}
$$

however, the magnitude of the resultant creep force cannot exceed the pure slip value that depends on the adhesion coefficient $\mu$ :

$$
T_{r, \lim }=\mu N
$$

Then a creep force saturation coefficient $\epsilon$ is defined according to the following modified Johnson-Vermeulen formulation [9]:

$$
\epsilon= \begin{cases}\frac{\mu N}{T_{r}}\left[\left(\frac{T_{r}}{\mu N}\right)-\frac{1}{3}\left(\frac{T_{r}}{\mu N}\right)^{2}+\frac{1}{27}\left(\frac{T_{r}}{\mu N}\right)^{3}\right] & \text { for } T_{r} \leq 3 \mu N, \\ \frac{\mu N}{T_{r}} & \text { for } T_{r}>3 \mu N .\end{cases}
$$

Finally the nonlinear creep force components are given by:

$$
\begin{aligned}
& T_{x}^{\prime}=\epsilon T_{x}, \\
& T_{y}^{\prime}=\epsilon T_{x} .
\end{aligned}
$$

This model is approximated since considers a constant adhesion coefficient $\mu$. More sophisticated models are present in the literature [19, 20], that consider the adhesion coefficient as a function of the sliding; they will be implemented in future versions of the software.

As an example, the results of a simulation of the vehicle dynamics on a curved track carried out through the Matlab software are shown. The results are obtained using the DIST method for the detection of the contact points. The main characteristics of the curve are summarized in Table 1.

Figures $18 \mathrm{a}$ and $\mathrm{b}$ show the curvature $K(s)$, the cant angle $\beta_{\mathrm{c}}(s)$ as a function of the curvilinear abscissa $s$. Figure $18 \mathrm{c}$ shows the track centerline on the plane $x_{f}, y_{f}$ : it is composed of an initial straight part $(50 \mathrm{~m})$, the curve, and a final straight part. 
Table 1 Characteristics of the curve

\begin{tabular}{lll}
\hline Laying angle & $\alpha_{p}$ & $1 / 20 \mathrm{rad}$ \\
Curvature & $K$ & $-1 / 1200 \mathrm{~m}^{-1}$ \\
Slope & $p$ & 0 \\
Cant angle & $\beta_{\mathrm{c}}$ & $60 / 1435 \mathrm{rad}$ \\
Train velocity & $V$ & $45 \mathrm{~m} / \mathrm{s}$ \\
Kinematic friction coefficient & $\mu_{c}$ & 0.2
\end{tabular}

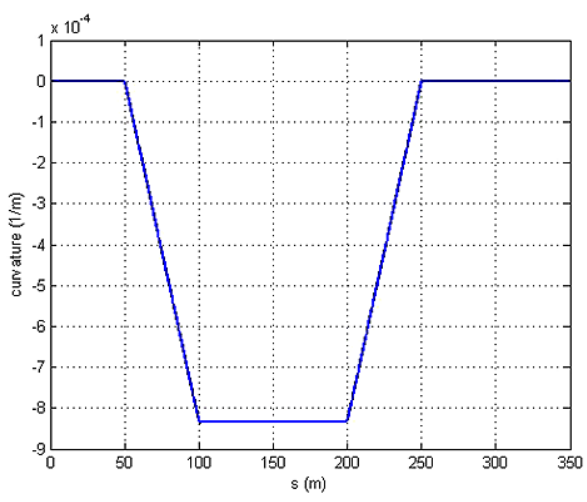

(a)

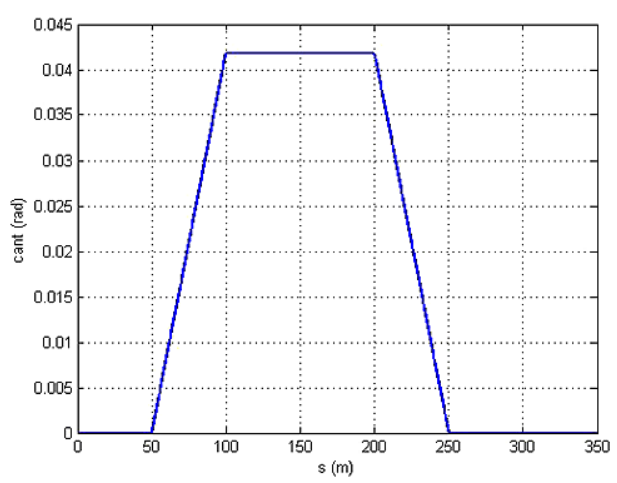

(b)

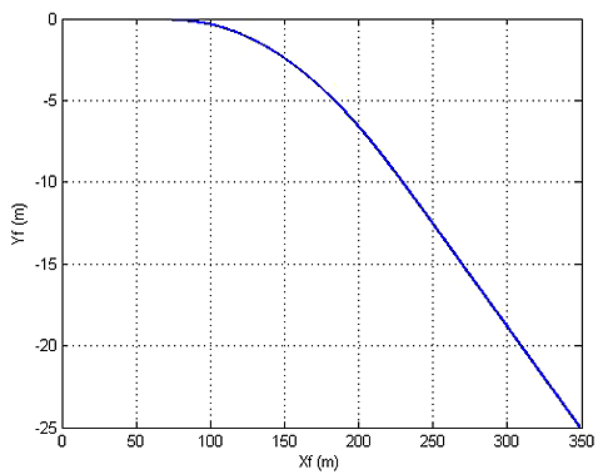

(c)

Fig. 18 Characteristics of the curve, (a) rail curvature, (b) cant angle, and (c) the track on the $x_{f} y_{f}$ plane

The multibody model allows to calculate all the kinematic and dynamic characteristic of each part of the model during the simulation; for brevity, only the following variables are considered:

- The lateral displacement $G_{y}$ of the center of mass of the first axle with respect to the track centerline (expressed in the auxiliary reference system). In the presented simulation, a right curve is considered (negative curvature) and, therefore, when the vehicle is on the curve the displacement is positive (Fig. 19).

- The positions of the contact points on the wheels and on the rails (expressed respectively in the local and in the auxiliary system). In order to clarify the results, the positions of these points have been plotted on two right cylinder surfaces having the same generative 
Fig. 19 Lateral displacement $G_{y}$ of the first axle

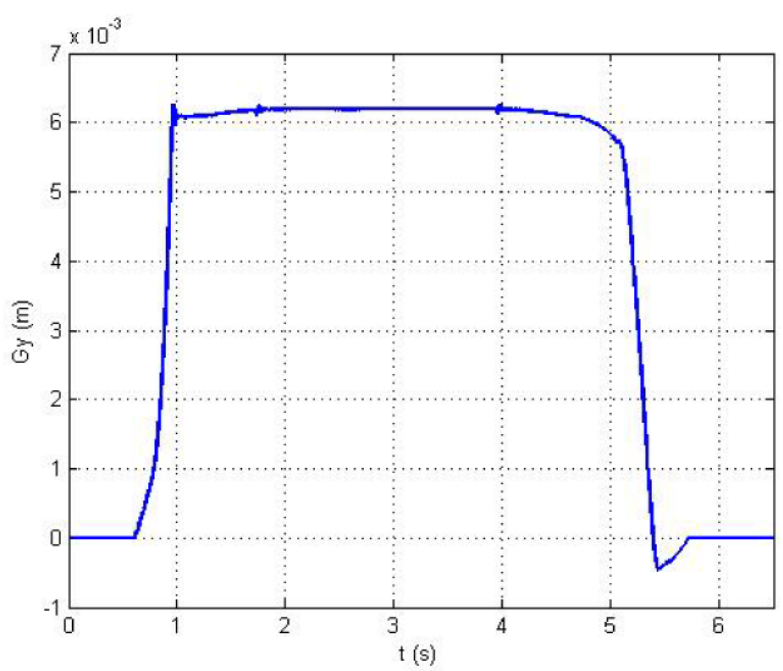

functions of the wheel and rail and a length equal to the distance traveled by the vehicle $(350 \mathrm{~m})$. As it can be observed during the curve, two contact points on the left side (Figs. 20a and c) and one on the right side (Figs. 20b and d) are present.

- The vertical contact forces $F_{z}$ on the wheels of the first axle (expressed in the auxiliary reference system) (Figs. 21a, b, and c). As it can be seen when the vehicle performs, the curve on the left wheel two contact points are present. When the second contact point appears, the vertical load on the first one decreases.

In the presented simulations, the DIST method has been implemented directly on the simulator (on line), then for each integration sample the contact points are evaluated as described in Sect. 2 as a function of the wheelset displacement, the contact point locations, the indentation values, and the wheelset velocity are used to evaluate the contact force, which allows to define the vehicle dynamics equations [11]. The proposed method is able to easily manage multiple contact points: The equation to be solved is the same both in case of single and multiple solution, the variable is the number of solutions.

\subsection{Performances of the proposed procedures}

The performances of the procedures for the detection of the contact points previously described have been compared among them and with those of other methods present in the literature $[10,11]$. Concerning the precision of the procedures, the following steps have been carried out:

1. Firstly, the DIST and the DIFF method have been compared: (43) and (62) have been solved numerically by means of a simple GRID algorithm (with grid resolution $\left.p_{g}=0.1 \mathrm{~mm}\right)$.

2. Then the DIFF method results has been compared with those obtained applying a simple multi-dimensional GRID method. This procedure consists essentially in the calculation of the difference $D\left(x_{w}, y_{w}\right)$ between the wheel and rail surfaces in the points defined by a fixed grid (with a resolution $p_{G}=1.0 \mathrm{~mm}$ on both the dimensions). As mentioned in the Introduction, this method is computationally inefficient, especially if the problem is multi-dimensional, but can be considered a reliable benchmark in terms of precision. 


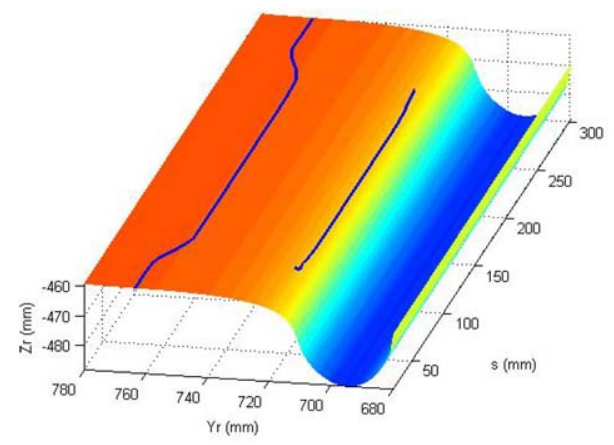

(a)

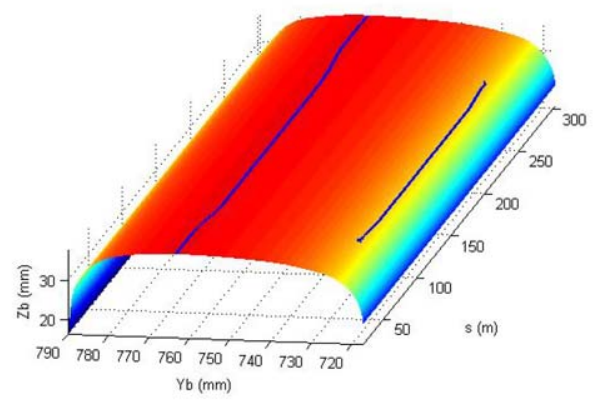

(c)

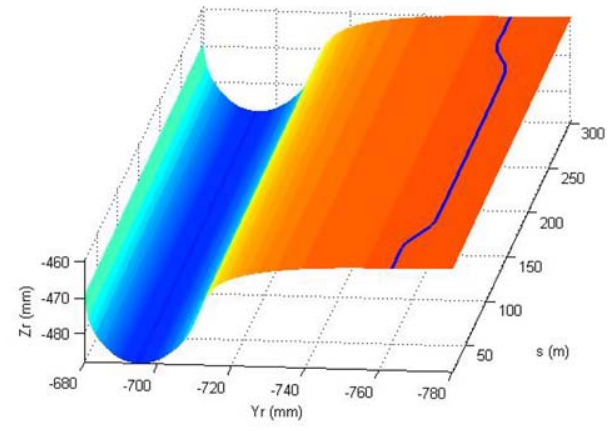

(b)

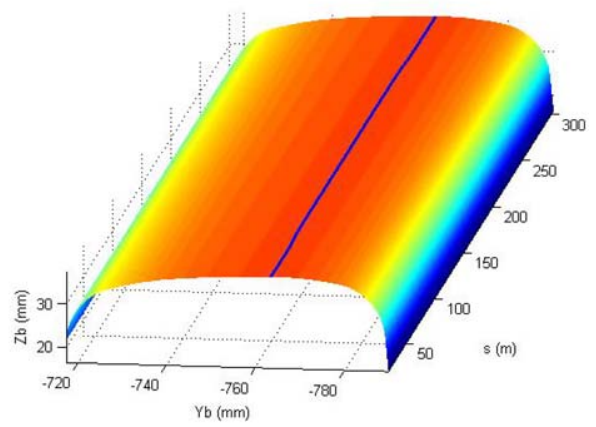

(d)

Fig. 20 Contact points on the wheels and on the rails, (a) left wheel, (b) right wheel, (c) left rail, and (d) right rail

3. The GRID method has been compared with the procedures described in the literature, based on numerical iterative algorithms applied to find the minima of the surface $D\left(x_{w}, y_{w}\right)$. In particular, the Compass Search method (indicated with CS) and the Simplex method (indicated with $S$ ) have been considered (with a tolerance of $0.1 \mathrm{~mm}$ for both the algorithms) $[12,13]$.

4. Finally, the DIFF method and the procedures based on the numerical iterative algorithms have been compared.

The comparison between the performance of the different methods for the evaluation of the contact point locations has been realized considering a set of relative wheelset/railway configurations composed of approximately $4.2 \times 10^{6}$ elements. The relative positions between wheel and rail have been obtained varying the parameters $G_{y}, G_{z}, \alpha$, and $\beta$ into the range summarized in Table 2 (the range has been chosen taking into account the symmetries of the problem).

All the configurations considered in the comparisons have been chosen in order to have in correspondence of the contact points, normal indentations $p_{n}$ (or $\tilde{p}_{n}$ ) physically acceptable: the bound $p_{n} \leq p_{l}=0.33 \mathrm{~mm}$ has been imposed. The limit value $p_{l}$ has been calculated through the Hertz theory hypothesizing a maximum normal load of $10^{5} \mathrm{~N}$ applied on a single contact point. 


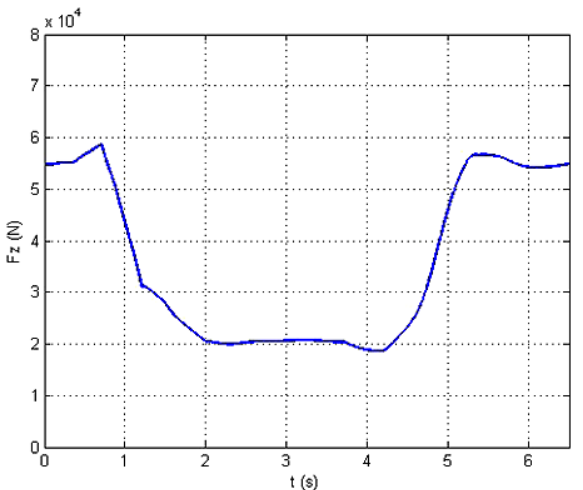

(a)

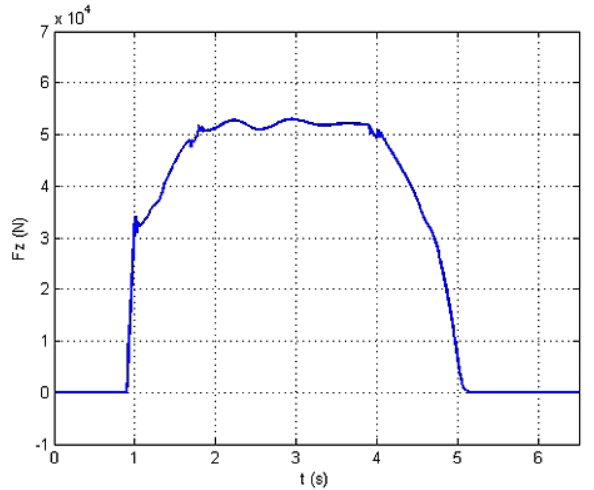

(b)

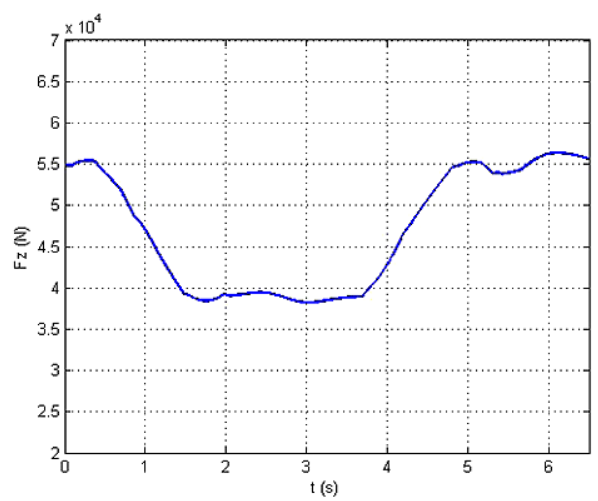

(c)

Fig. 21 Vertical contact forces, (a) vertical force $F_{z}$ on the first contact point of the left wheel, (b) vertical force $F_{z}$ on the second contact point of the left wheel, and (c) vertical force $F_{z}$ on the contact point of the right wheel

Table 2 Ranges of variability of the parameters

\begin{tabular}{ll}
\hline$G_{y}$ & $0 \mathrm{~mm} \div 10 \mathrm{~mm}$ \\
$G_{z}$ & $490 \mathrm{~mm} \div 510 \mathrm{~mm}$ \\
$\alpha$ & $0 \mathrm{rad} \div 0.01 \mathrm{rad}$ \\
$\beta$ & $-0.01 \mathrm{rad} \div 0.01 \mathrm{rad}$ \\
\hline
\end{tabular}

The algorithm used to compare two procedures for the determination of contact point locations detects the configurations in which the analyzed procedures evaluate a different number of contact points or the evaluated locations are not the same. To this purpose, a single wheel and the corresponding rail (indifferently on the left or on the right side) can be considered. The procedures used for the comparison are indicated with $A$ (benchmark) and $B$. For a generic wheel/rail configuration, $\mathbf{p}_{b i}^{C A}$ with $i=1,2, \ldots, N^{A}$ and $\mathbf{p}_{b j}^{C B}$ with $j=$ $1,2, \ldots, N^{B}$ indicate the contact points (on the rail surface) detected by the procedures $A$ and $B$, respectively, $N^{A}$ and $N^{B}$ are the numbers of contact points detected by the procedure $A$ and $B$, respectively. The number $E^{A B}$ of the errors associated to this configuration can be determined through the following algorithm: 
1. the vectors $\mathbf{v}_{A}=\mathbf{0}$ and $\mathbf{v}_{B}=\mathbf{0}$ where $\mathbf{v}_{A} \in \mathbb{R}^{N^{A}}$ and $\mathbf{v}_{B} \in \mathbb{R}^{N^{B}}$ are initialized

2. for $i=1,2, \ldots, N^{A}$

$$
\begin{aligned}
& \text { for } j=1,2, \ldots, N^{B} \\
& \quad \text { if }\left\|\mathbf{p}_{b i}^{C A}-\mathbf{p}_{b j}^{C B}\right\|_{2} \leq \text { toll and } v_{B}(j)=0 \\
& \quad \text { set } v_{A}(i)=1 \text { and } v_{B}(j)=1 \\
& \quad \text { break } \\
& \text { end } \\
& \text { end }
\end{aligned}
$$

end

3. set $n^{A}=N^{A}-\left\|\mathbf{v}_{A}\right\|_{2}^{2}$ and $n^{B}=N^{B}-\left\|\mathbf{v}_{B}\right\|_{2}^{2}$

4. $E^{A B}=\max \left(n^{A}, n^{B}\right)$

The elements of the vectors $\mathbf{v}_{A}$ and $\mathbf{v}_{B}$ are logical variables (their value can be 0 or 1 ). For example, the $j$-th element of the vector $\mathbf{v}_{A}$ is set equal to 1 if one of the contact points detected by the procedure $B$ is sufficiently near to the $j$-th contact point detected by the procedure $A$, in other terms, if the distance between the locations of the detected contact points is lower than a fixed value, identified by the variable toll. If the procedures $A$ and $B$ calculated the same number of contact points and their locations would be the same, $N^{A}=N^{B}$ and all the elements of the vectors $\mathbf{v}_{A}$ and $\mathbf{v}_{B}$ would be 1 . If the procedures $A$ and $B$ calculate a different number of contact points and/or if the distance between their locations overcome the fixed value toll, some of the elements of the vectors $\mathbf{v}_{A}$ and $\mathbf{v}_{B}$ are equal to 0 . For each procedure, the difference between the number of detected contact points and the number of contact points matching with the other procedure is defined with the variables $n^{A}$ and $n^{B}$.

For each wheel/rail configuration, the error $E^{A B}$ between the procedures $A$ and $B$ in the definition of the contact point locations is defined as the maximum between $n^{A}$ and $n^{B}$. Furthermore, $E^{A B}$ represents the sum of the undetected pairs of points $E_{1}^{A B}=\min \left(n^{A}, n^{B}\right)$ and of the points in excess $E_{2}^{A B}=\max \left(n^{A}, n^{B}\right)-\min \left(n^{A}, n^{B}\right)$. In order to evaluate the global algorithm performance and to analyze the configurations with multiple contact points, the error $E_{h k}^{A B}$ is defined as the number of errors between the procedures $A$ and $B$ in the $k$-th configuration with $h$ contact points. The partial errors $e_{h}^{A B}$ can then be defined as follows:

$$
e_{h}^{A B}=\frac{\sum_{k=1}^{T_{h}^{A}} E_{h k}^{A B}}{h T_{h}^{A}}, \quad h=1,2, \ldots, P^{A},
$$

where $T_{h}^{A}$ is the number of relative wheel-rail configurations with $h$ contact points and $P^{A}$ is the maximum number of contact points present on a single wheel. The global error $e^{A B}$ is then defined as:

$$
e^{A B}=\frac{\sum_{h=1}^{P^{A}} \sum_{k=1}^{T_{h}^{A}} E_{h k}^{A B}}{T^{A}},
$$

where $T^{A}=\sum_{h=1}^{P^{A}} h T_{h}^{A}$ is the total number of the contact points.

Concerning the comparison between the DIST and the DIFF method, Tables 3 and 4 summarizes the partial errors $e_{h}^{d D}$ and the global error $e^{d D}$ for different values of the laying angle $\alpha_{p}$. In this case, the DIST method has been chosen as benchmark with a tolerance toll $=2.0 \mathrm{~mm}$. 
Table 3 Comparison between the DIST (benchmark) and the DIFF methods, partial errors $e_{h}^{d D}$ and global error $e^{d D}$ $\left(\alpha_{p}=1 / 40\right)$

Table 4 Comparison between the DIST (benchmark) and the DIFF methods, partial errors $e_{h}^{d D}$ and global error $e^{d D}$ $\left(\alpha_{p}=1 / 20\right)$

Table 5 Comparison between the DIST (benchmark) and the DIFF methods, global error $e^{d D}$ $\left(\alpha_{p}=1 / 40\right)$

\begin{tabular}{ll}
\hline Error type & $\alpha_{p}=1 / 40$ \\
\hline$e_{1}^{d D}(\%)$ & 1.0 \\
$e_{2}^{d D}(\%)$ & 1.1 \\
$e^{d D}(\%)$ & 1.0 \\
\hline
\end{tabular}

\begin{tabular}{ll}
\hline Error type & $\alpha_{p}=1 / 20$ \\
\hline$e_{1}^{d D}(\%)$ & 0.3 \\
$e_{2}^{d D}(\%)$ & 2.3 \\
$e_{3}^{d D}(\%)$ & 1.6 \\
$e^{d D}(\%)$ & 0.9 \\
\hline
\end{tabular}

\begin{tabular}{llllllll}
\hline \multicolumn{7}{l}{ toll $(\mathrm{mm})$} \\
\cline { 3 - 7 } & & 0.5 & 1.0 & 1.5 & 2.0 & 2.5 & 3.0 \\
\hline$p_{n}$ & 0.30 & 2.3 & 1.9 & 1.3 & 1.1 & 1.0 & 0.9 \\
$(\mathrm{~mm})$ & 0.24 & 2.1 & 1.7 & 1.3 & 1.1 & 0.9 & 0.9 \\
& 0.18 & 2.1 & 1.5 & 1.1 & 1.0 & 0.9 & 0.9 \\
& 0.12 & 1.8 & 1.2 & 1.0 & 0.8 & 0.8 & 0.8 \\
& 0.06 & 1.0 & 0.6 & 0.4 & 0.4 & 0.4 & 0.4 \\
\hline
\end{tabular}

Table 6 Comparison between the DIST (benchmark) and the DIFF methods, global error $e^{d D}$ $\left(\alpha_{p}=1 / 20\right)$

\begin{tabular}{llllllll}
\hline \multicolumn{7}{l}{ toll $(\mathrm{mm})$} \\
\cline { 3 - 7 } & & 0.5 & 1.0 & 1.5 & 2.0 & 2.5 & 3.0 \\
\hline$p_{n}$ & 0.30 & 1.8 & 1.4 & 1.3 & 1.3 & 1.3 & 1.3 \\
$(\mathrm{~mm})$ & 0.24 & 1.3 & 1.1 & 1.0 & 1.0 & 1.0 & 1.0 \\
& 0.18 & 0.9 & 0.7 & 0.7 & 0.7 & 0.7 & 0.7 \\
& 0.12 & 0.5 & 0.4 & 0.4 & 0.4 & 0.4 & 0.4 \\
& 0.06 & 0.2 & 0.2 & 0.2 & 0.2 & 0.2 & 0.2 \\
\hline
\end{tabular}

Due to the wheel and rail geometries, when the laying rail angle is $\alpha_{p}=1 / 20$ configurations with one, two, and three contact points are present, when its value is $\alpha_{p}=1 / 40$, there are only configurations with one and two contact points. The comparison between DIST and DIFF shows a global error approximately equal to $1 \%$; its value substantially does not dependent on the laying angle.

Then the behavior of the global error $e^{d D}$ has been analyzed as a function of the tolerance toll and of the normal indentation $p_{n}$; both the standard values of the laying angle $\alpha_{p}$ have been considered (Tables 5 and 6). Also, in this case, the DIST method has been considered as the benchmark.

From the obtained results, it can be observed that

1. The global error $e^{d D}$ decreases if the tolerance toll increases. However, a bias error is present: this systematic error is due to the differences in the analytic formulation of the 
Table 7 Comparison between the DIFF (benchmark) and the GRID methods, global error $e^{\mathrm{DG}}$ $\left(\alpha_{p}=1 / 40,1 / 20\right)$

Table 8 Comparison between the GRID (benchmark) and the iterative (Compass Search and Simplex) methods, global errors $e^{\mathrm{GCS}}$ and $e^{\mathrm{GS}}\left(\alpha_{p}=1 / 40,1 / 20\right)$

Table 9 Comparison between the DIFF (benchmark) and the iterative (Compass Search and Simplex) methods, global errors $e^{\mathrm{DCS}}$ and $e^{\mathrm{DS}}\left(\alpha_{p}=1 / 40,1 / 20\right)$

\begin{tabular}{lll}
\hline Error type & $\alpha_{p}=1 / 40$ & $\alpha_{p}=1 / 20$ \\
\hline$e^{\mathrm{DG}}(\%)$ & 1.1 & 4.2 \\
\hline
\end{tabular}

\begin{tabular}{lll}
\hline Error type & $\alpha_{p}=1 / 40$ & $\alpha_{p}=1 / 20$ \\
\hline$e^{\mathrm{GCS}}(\%)$ & 3.2 & 5.9 \\
$e^{\mathrm{GS}}(\%)$ & 1.6 & 7.1 \\
\hline
\end{tabular}

\begin{tabular}{lll}
\hline Error type & $\alpha_{p}=1 / 40$ & $\alpha_{p}=1 / 20$ \\
\hline$e^{\mathrm{DCS}}(\%)$ & 5.0 & 10 \\
$e^{\mathrm{DS}}(\%)$ & 3.4 & 10 \\
\hline
\end{tabular}

two methods and to the different checks on the solutions: The first one evaluates the contact points as the points in which the distance between the body surfaces has a local maximum, while the second one minimizes the difference between the surface in the $z_{r}$ direction. The locations identified with the procedures are approximately the same if the indentation is small: the bias error decreases as the indentation decreases.

2. The global error $e^{d D}$ decreases if the normal indentation $p_{n}$ decreases.

As regards instead, the comparison between the DIFF and the GRID methods, the global error $e^{\mathrm{DG}}$ for the usual values of the laying angle $\alpha_{p}$ (Table 7) have been considered. In this case, the DIFF method has been chosen as benchmark with a tolerance toll $=2.0 \mathrm{~mm}$.

In the same way, both the CS and the S method have been compared to the GRID method (chosen as benchmark with tolerance toll $=4.0 \mathrm{~mm}$ ). Table 8 summarizes the values of the global errors $e^{\mathrm{GCS}}$ for the CS method and $e^{\mathrm{GS}}$ for the S method, with $\alpha_{p}=1 / 40$ and $\alpha_{p}=1 / 20$.

As regards finally, the comparison between the procedures based on the numerical iterative algorithms and the DIFF method (chosen as benchmark with tolerance toll $=2.0 \mathrm{~mm}$ ), the global errors $e^{\mathrm{DCS}}$ and $e^{\mathrm{DS}}$ for the standard values of the laying angle $\alpha_{p}$ are summarized in Table 9.

The performances of the various procedures, moreover have been compared among them in terms of computation times. Figure 22 shows the mean time required to evaluate the contact points in a generic relative wheel-rail configurations (the corresponding values are summarized in Table 10). The described performance have been obtained with a processor Intel Pentium $4(3.0 \mathrm{GHz})$.

On the basis of the described results, the following considerations can be made:

1. The performances of the DIST and the DIFF methods are similar in terms of precision and computation times.

2. The semianalytic procedures are reliable as regards precision.

3. The DIST and the DIFF methods are faster and more accurate than the procedures based on the numerical iterative algorithms. 
Table 10 Computation times

\begin{tabular}{ll}
\hline Method & Time (s) \\
\hline GRID & 9.3 \\
S & 0.255 \\
CS & 0.105 \\
DIST & 0.045 \\
DIFF & 0.037 \\
\hline
\end{tabular}

Fig. 22 Comparison among the computation times

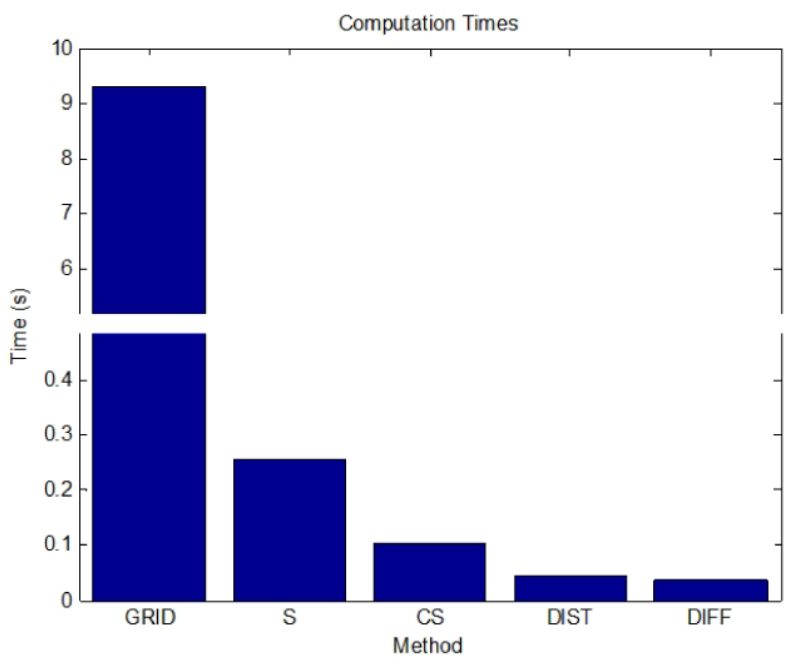

\section{Conclusions}

In this work, two semianalytic methods for the detection of the wheel-rail contact points (the DIST and the DIFF methods) are presented. Both the procedures consider the wheel and the rail as two mathematical surfaces whose analytic expression is known. The DIST procedure is based on the idea that the contact points minimize the distance between the surfaces and is equivalent to solve an algebraic 4D-system. The DIFF method instead is based on the idea that the contact points minimize the difference between the surfaces and is equivalent to solve an algebraic 2D-system, it is based on an approximation whose consequences in terms of reliability has been verified with a series of tests.

In both cases, the original problem has been reduced analytically to an equation with one scalar unknown that is then solved numerically. Since the problem dimension is one, even elementary noniterative algorithms have shown to be efficient and reliable.

Subsequently, the performances of the described procedures have been compared among them and with those of the methods present in the literature. The GRID method and other procedures based on numerical iterative algorithms (like the Compass Search algorithm and the Simplex algorithm) have been considered. The comparison has been carried out in terms of precision and computation times.

The developed procedures (named DIST and DIFF methods) have similar performances in terms of precision and computation times, both of them are reliable as regards the precision and are more accurate and more efficient than the procedures based on the numerical iterative algorithms. 
The reduced computational times allow to overcome the usual off-line implementation, in which the contact points are calculated independently from the vehicle dynamics model and used to build lookup tables used during the simulations and to realize an online implementation (the procedure can be implemented directly in the multibody model).

\section{References}

1. Shabana, A.A., Sany, J.R.: An augmented formulation for mechanical systems with non-generalized coordinates: application to rigid body contact problems. Nonlinear Dyn. 24, 183-204 (2001)

2. Shabana, A.A., Zaazaa, K.E., Escalona, J.L., Sanyc, J.R.: Development of elastic force model for wheel/rail contact problems. J. Sound Vib. 269, 295-325 (2004)

3. Shabana, A.A., Tobaa, M., SugiYama, H., Zaazaa, K.E.: On the computer formulations of the wheel/rail contact problem. Nonlinear Dyn. 40, 169-193 (2005)

4. Shabana, A.A., Sany, J.R.: A survey of rail vehicle track simulations and flexible multibody dynamics. Nonlinear Dyn. 26, 179-210 (2001)

5. Shabana, A.A., Berzeri, M., Sany, J.R.: Numerical procedure for the simulation of wheel/rail contact dynamics. Trans. Am. Soc. Mech. Eng. 123, 168-178 (2001)

6. Pombo, J., Ambrosio, J.: Dynamic analysis of a railway vehicle in real operation conditions using a new wheel-rail contact detection model. Int. J. Veh. Syst. Model. Test. 1(1/2/3), 79-105 (2005)

7. Rulka, W., Pankiewicz, E.: MBS approach to generate equations of motions for hil-simulations in vehicle dynamics. Multibody Syst. Dyn. 14, 367-386 (2005)

8. Kik, W., Moelle, D.: Implementation of the wheel-rail element in ADAMS/Rail Version 10.1. In: 5th ADAMS/Rail Users' Conference, Haarleem (2000)

9. Dukkipati, R.V., Amyot, J.R.: Computer Aided Simulation in Railway Dynamics. Dekker, New York (1988)

10. Malvezzi, M., Meli, E., Papini, S., Pugi, L.: Parametric models of railway systems for real-time applications. Multibody dynamics, Milano, Italy (2007)

11. Auciello, J., Malvezzi, M., Meli, E., Papini, S., Pugi, L., Rindi, A.: Multibody models of railway vehicles for real-time systems. In: XVIII Congresso AIMETA, Brescia, Italy (2007)

12. Kolda, T.G., Lewis, R.M., Torczon, V.: Optimization by direct search new perspectives on some classical and modern methods. SIAM Rev. 45(3), 385-482 (2003)

13. Nelder, J.A., Mead, R.: A simplex method for function minimization. Comput. J. 7, 308-313 (1965)

14. do Carmo, M.P.: Differential Geometry of Curves and Surface. Prentice Hall, Englewood Cliffs (1976)

15. Esveld, C.: Modern Railway Track, 2nd edn. Delft University of Technology, Delft (2001)

16. Iwnicki, S.: The Manchester Benchmarks for Rail Vehicle Simulators. Swets \& Zeitlinger, Lisse (1999) (ISBN 9026515510 )

17. Kalker, J.J.: Three-Dimensional Elastic Bodies in Rolling Contact. Kluwer Academic, Dordrecht (1990)

18. Vollebregt, E.A.H., Kalker, J.J., Wang, G.: CONTACT 93 Users Manual. VORtech Computing, Industrial and Scientific Computing, July 1992. Revised March 1994

19. Polach, O.: Creep forces in simulations of traction vehicles running on adhesion limit. Wear 258, 9921000 (2005)

20. Polach, O.: Influence of locomotive tractive effort on the forces between wheel and rail. Veh. Syst. Dyn. 35, 7-22 (2001) 